\title{
Simulation of Electromagnetic Fields Generated by Overhead Power Lines and Railroad Traction Networks
}

\author{
Natalya V. Buyakova ${ }^{1,}$ Vasiliy P. Zakaryukin ${ }^{2}$ Andrey V. Kryukov ${ }^{*}{ }^{3,4}$, Dmitriy A. Seredkin ${ }^{3}$
}

\author{
${ }^{1}$ Angarsk State Technical University, Angarsk, Russia \\ ${ }^{2}$ Smart Grid LLC, Irkutsk, Russia \\ ${ }^{3}$ Irkutsk State Transport University, Irkutsk, Russia \\ ${ }^{4}$ Irkutsk National Research Technical University, Irkutsk, Russia
}

\begin{abstract}
The establishment of smart grids requires special attention to the safety of power industry facilities and the reduction in their negative impact on personnel and the environment. A significant factor of such an impact is the considerable intensities of electromagnetic field (EMF) generated by such facilities. In the context of power industry digitalization, the development of methods and tools for EMF digital simulation is needed to ensure the electromagnetic safety of the service personnel. Computer models designed to adequately determine the EMF generated by overhead power lines (OPL) can be implemented based on the methods and tools created to determine operating parameters of electric power systems in phase coordinates developed at Irkutsk State Transport University. The technique of electromagnetic safety analysis is implemented based on the proposed approach and has the following features: a systems approach, which is the possibility of simulating electromagnetic fields in terms of properties and characteristics of a complex electric power system; versatility, which allows simulating power lines and traction networks of various designs; appropriateness to the environment achieved by considering the profile of the underlying surface, underground utilities, and artificial structures of rail transport, such as galleries, bridges, and tunnels; comprehensiveness, which is provided by combining the computation of operating parameters and the determination of EMF intensities. The paper describes methods and algorithms developed to determine the intensities of electromagnetic
\end{abstract}

\footnotetext{
${ }^{*}$ Corresponding author.

E-mail: and kryukov@mail.ru
}

http://dx.doi.org/10.38028/esr.2021.02.0007

Received June 26, 2021. Revised July 01, 2021.

Accepted July 13, 2021. Available online July 23, 2021.

This is an open access article under a Creative Commons Attribution-NonCommercial 4.0 International License.

(C) 2021 ESI SB RAS and authors. All rights reserved. fields generated by multi-conductor power lines at fundamental and higher harmonics frequencies. A technique for the EMF determination at the points of OPL orthogonal crossing is also presented.

Index Terms: modeling, electromagnetic field, power transmission line, traction network.

\section{INTRODUCTION}

Electric power facilities generate electromagnetic fields (EMFs) of fundamental and higher harmonic frequencies $[1,2]$, which are among the main factors that determine the electromagnetic safety conditions [3-17]. High-intensity electromagnetic fields can generate interference causing malfunctioning of electrical and electronic devices and result in serious accidents when one operates at tripped power lines due to the impact of induced voltage on personnel.

Methods and tools for simulation of operating conditions in phase coordinates developed at Irkutsk State Transport University (ISTrU) [18] allow simultaneous computations of EMF for multi-conductor power lines [4] and determination of operating conditions of the electric power system (EPS) or traction power supply system (TPSS), being its part. In this case, the line at issue is considered inseparably with a complex EPS or TPSS. Simultaneous computation of operating conditions and generated EMFs enables the systems approach to the electromagnetic environment analysis. Its distinct feature is the possibility of EMF simulation with proper consideration of all properties and characteristics of the complex TPSS and EPS. Advantages of the method proposed are, first of all, the possibility for simulation modeling $[4,18]$ of trains operation in the TPSS under study and determination of EMF intensity dynamics, and secondly, due consideration of factors that affect the EMF intensity levels, including [4]:

- unevenness of the underlying surface caused by embankments, depressions, slopes, and passenger platforms;

- metal railroad cars and cisterns on tracks that 
significantly affect spatial distribution patterns of EMF intensities;

- $\quad$ earthed extended metal objects (pipelines, cable lines with earthed coatings, and earthed ropes) that also change the EMF distribution pattern.

The methods and tools developed at ISTrU for modeling the TPSS operation and the Fazonord software implemented on their basis allow using several hundred wires in the models [18]. This makes it possible to simulate the embankments, depressions, railroad cars, and cisterns with a set of wires earthed at one end and located so as to make the distance between the wires significantly shorter than the distance to the observation point. This technology also allows calculation of EMF in artificial railroad transport structures, i.e., tunnels, galleries, bridges.

The proposed approach underlies the technique of electromagnetic safety analysis that has the following characteristics:

- systems approach, which manifests itself through the possibility of modeling electromagnetic fields by factoring in the properties and characteristics of a complex TPSS and a supply EPS;

- universality, which ensures modeling of power transmission lines and traction networks of various designs;

- consideration of the environment via a thorough analysis of underlying surface, underground utilities, and track structures, including galleries, bridges, and tunnels;

- integrity ensured by combining the computation of operating conditions and the determination of EMF intensities.

\section{MODELING OF OPERATING CONDITIONS IN PHASE COORDINATES}

In a more generalized option, the modeling of electrical network operation can be represented as the following functional relationship:

$$
\mathbf{A}: \mathbf{D} \Rightarrow \mathbf{X}
$$

where $\mathbf{A}$ - the nonlinear operator; $\mathbf{D}=\mathbf{S} \cup \mathbf{Y}$ - the vector of source data; $\mathrm{X}$ - the vector of operating parameters; $\mathbf{S}$ - the set of data describing the structure and parameters of EPS (TPSS) components; Y - parameters characterizing generators and loads.

The system of steady-state equations (nonlinear in a general case) is generated by transformation of (1):

$$
\mathbf{F}(\mathbf{X}, \mathbf{Y})=0,
$$

where $\mathbf{X}$ - the vector formed from node voltage components in Cartesian $\left(U_{k}^{\prime}, U_{k}^{\prime \prime}\right)$ or polar $\left(U_{k}, \delta_{k}\right)$ coordinates; $\mathbf{Y}$ the vector that includes active and reactive power of generators and loads.

Technology of simulating the EPS operating conditions in phase coordinates [18] serves as the basis for models and methods proposed in this work. The EPS (TPSS) modeling methods rely on lattice-type equivalent circuits (LEC) with fully connected topology. The following formalized definition can be written for LEC:

$$
\text { TEC : hub } \bigcup c o n, \forall i, j \subset h u b \rightarrow \text { con }_{i, j} \subset c o n,
$$

where $T E C$ - stands for lattice-type equivalent circuit; $h u b$ - a set of LEC nodes; con - a set of LEC branches.

The basic elements that constitute the EPS three-phase - one-phase network supplying traction power systems can be subdivided into two groups:

- electricity transport elements, i.e., overhead power lines and cable lines, conductors, traction networks;

- conversion elements, i.e., transformers of various designs.

The above devices can be generally considered as static multi-wire elements, which can be represented as a set of wires or windings with inductive couplings (Fig. 1). Simulation of power sources, electrical loads and elements employed to control EPS operating conditions are analyzed in detail in $[18,19]$. Adequate assessment of electromagnetic safety conditions requires, apart from EMF to be determined for particular operation situations, the construction of time relationships between the intensities of electric $\dot{E}=\dot{E}(t)$ and magnetic $\dot{H}=\dot{H}(t)$ fields. These tasks can be accomplished based on simulation modeling. In this case, the concept of instantaneous diagrams is used and dynamic model is reduced to a set of static diagrams. The simulation procedure involves dividing the interval under study into smaller intervals, within which the operating parameters are assumed to be invariable. The analysis of experimental measurements of operating parameters in real TPSSs, and computer simulation results indicate that such an assumption is acceptable and does not introduce a significant error to computation results.

The development of a simulation model of the mainline railroad power system requires the construction of models of the EPS and TPSS elements with an algorithm of their interaction and includes the following stages:

- modeling of train operation schedule;

- generation of instantaneous diagrams and calculation of operating parameters for each of them;

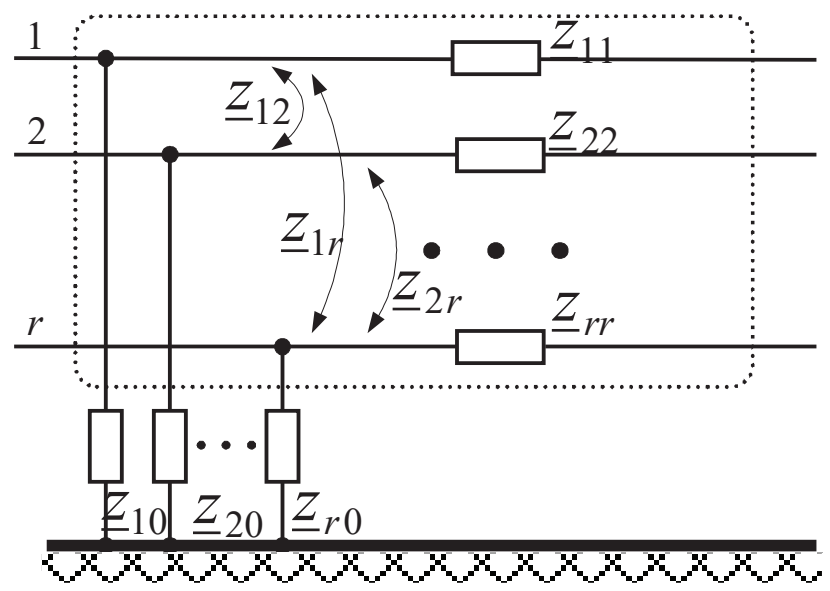

Fig. 1. The diagram of static multi-wire element. 
- determination of integral values of simulation modeling. At each modeling interval $\Delta t$, the following system of non-linear equations that define steady-state of the respective instantaneous diagram is solved:

$$
\mathbf{F}\left(\mathbf{X}_{k}, \mathbf{Y}_{k}\right)=0 \text {, }
$$

where $\mathbf{X}_{k}, \mathbf{Y}_{k}$ - values of vectors $\mathbf{X}, \mathbf{Y}$ for the $k$-th instantaneous diagram.

Modeling of moving traction loads is based on traffic schedule, which relates train location coordinate with time. Traction load values are determined based on traction computations or experiments. Traction loads are normally set by values of currents, although such an approach insufficiently adequately defines the physics of running processes. The train driver shall follow the specified traffic schedule. Thus, a change in voltage at current collecting equipment causes the need to adjust the electric locomotive current to maintain the necessary traction effort. Consequently, setting traction load with power consumed is a more appropriate approach that reflects energy conservation law. Software tools developed to determine traction loads obtain traction currents under rated voltage of the current collecting equipment, consequently, there is no problem in their translation to consumed power.

Railroad AC power system can be divided into the following segments:

- three-phase EPS that performs the external power supply function;

- one-phase traction power supply system;

- power supply areas of non-traction and non-transport consumers, including transmission lines of particular design following the pattern "wire - rail" and "two wires - rail."

The railroad power supply systems (RPSS) have longitudinal and transversal asymmetry of parameters due to the single-phase traction networks and devices of transverse and series capacitive compensation. This asymmetry affects the operation of the external power supply system and power supply areas.

The operating conditions for integrated systems of traction and external power supply are calculated by successively determining the operating conditions for some instantaneous diagrams of train operation. The instantaneous diagram requires the following data:

- a connection diagram of stationary elements with data on their parameters, as well as loads and generation of the RPSS stationary part; the location of electrical traction loads at the considered time instant, which is determined by train operation schedule; location of trains determines parameters of the system's changing part that includes the traction network sections;

- loads created by trains at the time instant at issue; these loads are determined based on traction loads or experimental rides.

The train schedule is used to determine the train location. The train location determines the lengths of the current sections of the traction network between its discontinuities, i.e., between neighboring trains or between trains and stationary nodes that include connection points of traction network feeders, sectioning points, parallel connection points, neutral sections, switching to another number of tracks or another suspension type.

\section{EMF SIMULATION IN PHASE COORDINATES}

After determining the instantaneous diagram operation by solving the set of equations (2), we can calculate the intensities of electromagnetic fields generated by any of the multi-wire systems, which are part of the simulated system. With the vertically upward direction of the Y-axis of the Cartesian coordinate system and the $\mathrm{X}$-axis perpendicular to the railroad or power line axis so that the $\mathrm{Z}$-axis is oriented in a negative direction of the wire current, the components of the electric field intensity of the system of $\mathrm{N}$ wires at the point with coordinates $(\mathrm{x}, \mathrm{y})$ are determined using the following formulas:

$$
\begin{aligned}
& \dot{E}_{Y}=-\frac{1}{\pi \varepsilon_{0}} \sum_{i=1}^{N} \dot{\tau}_{i} \frac{y_{i}\left[\left(x-x_{i}\right)^{2}-y^{2}+y_{i}^{2}\right]}{\xi_{i}} ; \\
& \dot{E}_{X}=\frac{2}{\pi \varepsilon_{0}} \sum_{i=1}^{N} \dot{\tau}_{i} \frac{\left(x-x_{i}\right) y y_{i}}{\xi_{i}}
\end{aligned}
$$

where $\quad \xi_{i}=\left[\left(x-x_{i}\right)^{2}+\left(y+y_{i}\right)^{2}\right]\left[\left(x-x_{i}\right)^{2}+\left(y-y_{i}\right)^{2}\right]$; $\dot{\tau}_{i}-$ charge of wire $i$ per length unit, which is determined from the first group of Maxwell's formulas $\dot{\mathbf{T}}=\mathbf{A}^{-1} \cdot \dot{\mathbf{U}}$.

Here $\quad \dot{\mathbf{U}}=\left[\begin{array}{lll}\dot{U}_{1} & \ldots & \dot{U}_{N}\end{array}\right]^{T}-$ column-vector of wire voltages relative to earth; $\dot{\mathbf{T}}=\left[\begin{array}{lll}\dot{\tau}_{1} & \ldots & \dot{\tau}_{N}\end{array}\right]^{T}-$ columnvector of wire charges, $\mathbf{A}$ - symmetric matrix of potential coefficients, in which

$$
\begin{gathered}
\alpha_{i i}=\frac{1}{2 \pi \varepsilon_{0}} \ln \frac{2 y_{i}}{r_{i}}, \\
\alpha_{i j}=\frac{1}{2 \pi \varepsilon_{0}} \ln \frac{\sqrt{\left(x_{i}-x_{j}\right)^{2}+\left(y_{i}+y_{j}\right)^{2}}}{\sqrt{\left(x_{i}-x_{j}\right)^{2}+\left(y_{i}-y_{j}\right)^{2}}},
\end{gathered}
$$

where $x_{i}, y_{i}$ - the coordinates of wire $i$ above the ground ( $\mathrm{y}=0$ corresponds to the flat earth surface), $r_{i}$ - radius of wire $i ; \varepsilon_{0}-$ electrical constant.

After transition from complex effective values of components $\dot{E}_{X}$ and $\dot{E}_{Y}$ to time dependences, one can obtain parametric equations for locus of the electrical field intensity vector:

$$
\begin{aligned}
& E_{x}(t)=\sqrt{2} E_{X} \sin \left(\omega t+\varphi_{X}\right) ; \\
& E_{y}(t)=\sqrt{2} E_{Y} \sin \left(\omega t+\varphi_{Y}\right),
\end{aligned}
$$

where the factor $\sqrt{2}$ is required because computations are based on effective values; $\omega=314 \mathrm{rad} / \mathrm{s}$.

The field intensity reaches its maximum value $E_{\max }$ at time instants defined by the following equation:

$$
t_{\max }=\frac{1}{2 \omega} \operatorname{Arctg}\left(\frac{E_{X}^{2} \sin 2 \varphi_{X}+E_{Y}^{2} \sin 2 \varphi_{Y}}{E_{X}^{2} \cos 2 \varphi_{X}+E_{Y}^{2} \cos 2 \varphi_{Y}}\right) .
$$


One of arctangent values is chosen when the second derivative has a negative value:

$$
E_{X}^{2} \cos 2\left(\omega t_{\max }+\varphi_{X}\right)+E_{Y}^{2} \cos 2\left(\omega t_{\max }+\varphi_{Y}\right)<0 .
$$

The effective value of field intensity for some direction $\psi$, measured from the $X$-axis positive direction is equal to

$$
\begin{gathered}
E_{\psi}=\sqrt{E_{X}^{2} \cos ^{2} \psi+E_{Y}^{2} \sin ^{2} \psi+D_{E}} ; \\
D_{E}=2 E_{X} E_{Y} \sin \psi \cos \psi \cos \left(\varphi_{X}-\varphi_{Y}\right) .
\end{gathered}
$$

Intensity extreme value is calculated using the following formula:

$$
\begin{gathered}
E_{\Psi E}=\left[\frac{\left(E_{X}^{2}+E_{Y}^{2}\right)^{2}}{2} \pm \frac{\sqrt{D_{\Psi E}}}{2}\right]^{\frac{1}{2}} ; \\
D_{\Psi E}=\left(E_{X}^{2}+E_{Y}^{2}\right)^{2}-4 E_{X}^{2} E_{Y}^{2} \sin ^{2}\left(\varphi_{X}-\varphi_{Y}\right) .
\end{gathered}
$$

In this case, plus sign corresponds to the maximum, and the minus sign corresponds to the minimum.

This formula is given in [21], where the authors point out that in the calculation of field near the ground surface, the error of simple quadratic summation

$$
E=\sqrt{E_{X}^{2}+E_{Y}^{2}}
$$

usually does not exceed $10 \%$ towards increase above the effective value maximum.

The horizontal and vertical components of the intensity of the magnetic field generated by all conductors are calculated using the following formulas:

$$
\begin{aligned}
& \dot{H}_{X}=\frac{1}{2 \pi} \sum_{i=1}^{N} \dot{I}_{i} \frac{y-y_{i}}{\left(x_{i}-x\right)^{2}+\left(y_{i}-y\right)^{2}} ; \\
& \dot{H}_{Y}=-\frac{1}{2 \pi} \sum_{i=1}^{N} \dot{I}_{i} \frac{x-x_{i}}{\left(x_{i}-x\right)^{2}+\left(y_{i}-y\right)^{2}} .
\end{aligned}
$$

The determination of the electric and magnetic field intensities involves the calculation of the RPSS operating conditions, the determination of charges and currents of wires, including earthed ones, and the components $\dot{E}_{X}, \dot{E}_{Y}, \dot{H}_{X}, \dot{H}_{Y}$. The described technique is implemented in the Fazonord software [18]. In this case, electromagnetic field intensities can be determined both for an individual instantaneous diagram and for their set, which determines the change in EMF intensities over time.

Such a problem statement significantly simplifies the computation of EMF intensities. Indeed, the traditional formulation of this problem requires solving differential equations in partial derivatives. Traditional methods considerably complicate the problem-solving procedure, especially when there is a need to factor in the underlying surface inhomogeneities (embankments, slopes, depressions) and the extended conductive facilities (artificial structures of railroad transport, metal cars and cisterns, underground pipelines, and others). The use of sets of grounded wires as part of the corresponding multiwire element for simulation of roadbed inhomogeneity and conductive facilities allows the use of the proposed technique to determine EMF without additional complications and modifications, given the external environment [4].

Computations of electric and magnetic field intensities under the proposed technique can be referred to as integral computation methods with a distribution of charges on fictitious earthed conductors located on the surface of nonplanar (but parallel to the plane) ground or the surface of the conductive boundary of an artificial structure. In contrast to the integral methods used, in this technique, the charges on the earthed conductors are found through the calculations of operating conditions in phase coordinates. After the operating conditions of a system that includes a multi-wire element are calculated, it is possible to determine the wire charges per unit of length.

Since the resistances of multi-wire power lines are calculated using the height of the equivalent wire, which is below the point of the wire suspension on the pole by twothirds of the slack, the computation will give some averaged electric field intensity value throughout span length. At a small (about two or three meters) height of the observation point location, the actual intensity in the middle of the span will be somewhat higher than the calculated one, while at the pole, it will be less than the calculated one.

These differences, however, are relatively small and are usually overarched by the idealization of the considered facility and errors of source data.

The EMF active power flux density can be determined using the formula [4]

$$
\begin{gathered}
\Pi_{0}=\frac{1}{2}\left[\Pi_{01}-\Pi_{02}\right] ; \\
\Pi_{01}=E_{m x} H_{m y} \cos \left(\psi_{E x}-\psi_{H y}\right) ; \\
\Pi_{02}=E_{m y} H_{m x} \cos \left(\psi_{E y}-\psi_{H x}\right) .
\end{gathered}
$$

Thus, after computing the components $\dot{E}_{X}, \dot{E}_{Y}, \dot{H}_{X}, \dot{H}_{Y}$, electromagnetic energy flux density determined by the Poynting vector can be calculated.

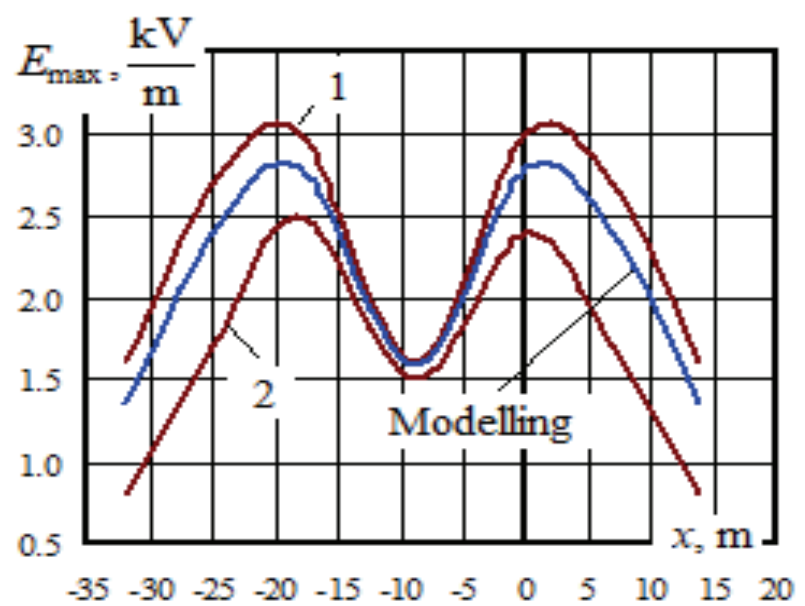

Fig. 2. Comparison of experimental and calculated data: 1, 2boundaries of the experimental data scatter area. 


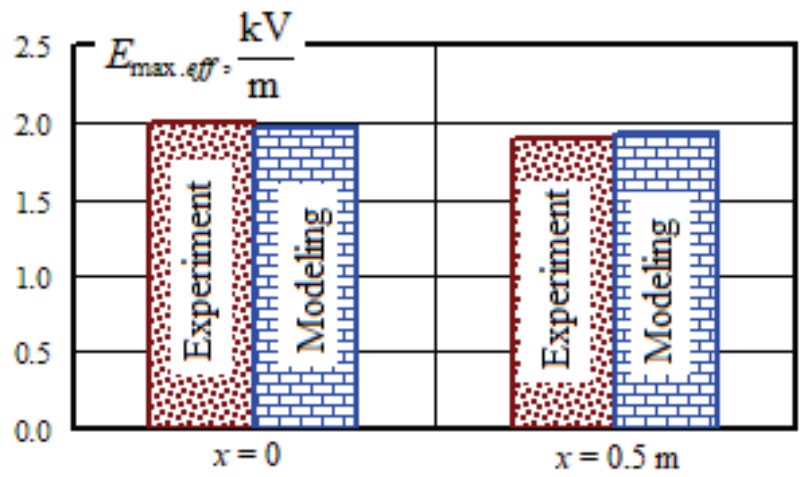

Fig. 3. Calculated and experimental values of the electric field intensity of the overhead system.

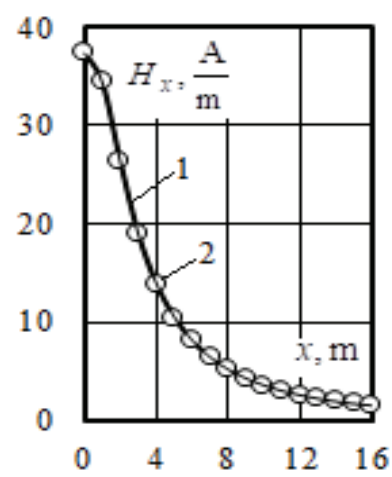

a)

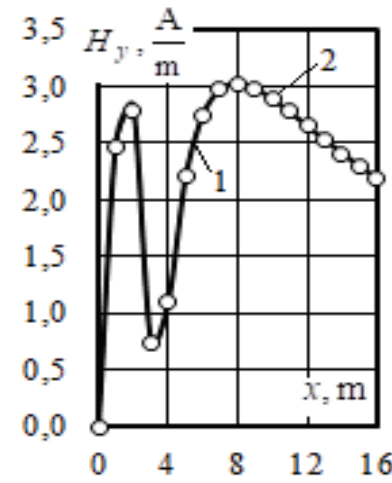

b)

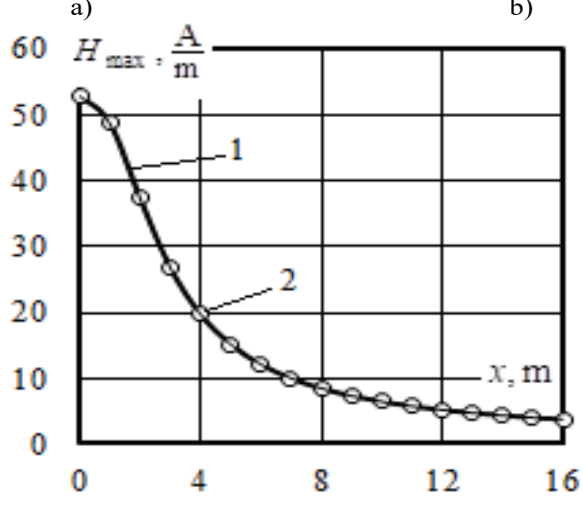

c)

Fig. 4. Comparison results.

\section{VERIFICATION OF THE SIMULATION ADEQUACY}

Experimental verification of the technique was carried out by comparing the calculation results for the intensity of electric field generated by $220 \mathrm{kV}$ transmission line with the experimental data obtained in [20]. The results of measurements and computations indicating the acceptable accuracy are shown in Fig. 2.

Fig. 3 shows the results of calculations and experimental studies on the electric field of the overhead system [3]. The results obtained indicate that the discrepancy between the experimental and calculated data does not exceed $2 \%$.

Experimental verification of the adequacy of calculation of the overhead system magnetic field is problematic because it is difficult to obtain source data for simulation of the RPSS operating conditions. Such information can be obtained only in the future, when the satellite system is employed to locate the trains in space. It is also difficult to restore the overhead power line conditions as there are no means for measuring magnitudes and phases of currents flowing through its wires.

Such measurements will also become possible only when electric power systems are equipped with devices for synchronized measurements of phasors (PMU-WAMS) on a large scale.

Therefore, the verification of adequacy of the magnetic field intensity calculations relied on the comparison with the results of analytical calculations (Fig. 4). The findings show a good agreement between the data of analytical computations and simulation based on the proposed technique.

To additionally verify the simulation adequacy, 50 $\mathrm{Hz}$ EMF intensities were measured, and comparative calculations for a $110 \mathrm{kV}$ double-circuit line with a lightning protection cable located within the city were performed. These measurements were made between two towers. The heights of the wire suspension above the measurement site were $19 \mathrm{~m}$ (ground wire), $16 \mathrm{~m}, 13 \mathrm{~m}$, and $10 \mathrm{~m}$ (phase wires). A $110 \mathrm{kV}$ single-circuit dead-end tap is connected to a line at the closest tower to the measurement site. At a distance of $10 \mathrm{~m}$ from the power transmission line with a slight decrease in the earth surface, there is a metal fence of the garage cooperative, which can have a significant effect on the electric field.

According to the data of measurements of operating parameters of a double-circuit overhead power line (OPL) located close to the branch line, the power flow of the first circuit was about $9-j 12 \mathrm{MV} \cdot \mathrm{A}$ (the minus sign corresponds to the accepted positive direction of the power flow of the branch line); the power flow on the second circuit was 6 $-j 9 \mathrm{MV} \cdot \mathrm{A}$; the branch line consumes $6+j 3 \mathrm{MV} \cdot \mathrm{A}$. To correctly factor in the multi-wire system with the facilities affecting EMF (metallic fence), its model, including 19 wires, was implemented in the Fazonord software.

Phase voltages were assumed symmetrical, equal to 65 $\mathrm{kV}$ for a more loaded circuit and $66 \mathrm{kV}$ for a less loaded one. In the calculation of operating conditions, the above loads symmetrically distributed among phases resulted in 77 A currents for the left-hand circuit wires, $54 \mathrm{~A}$ for the right-hand circuit wires, and $34 \mathrm{~A}$ for the branch line.

According to the calculation of operating conditions, the value of the ground wire current was of the order of 0.1 A. Thus, while significantly changing the electric field, the ground wire has almost no effect on the magnetic field.

Experimental measurements of electromagnetic field intensities, performed with the P3-50 device, are represented by three groups of values obtained at different times; the directions of coordinate axes were assumed the same as in the computation technique. The origin of coordinates is under the OPL center in the middle of the span on the ground surface. 


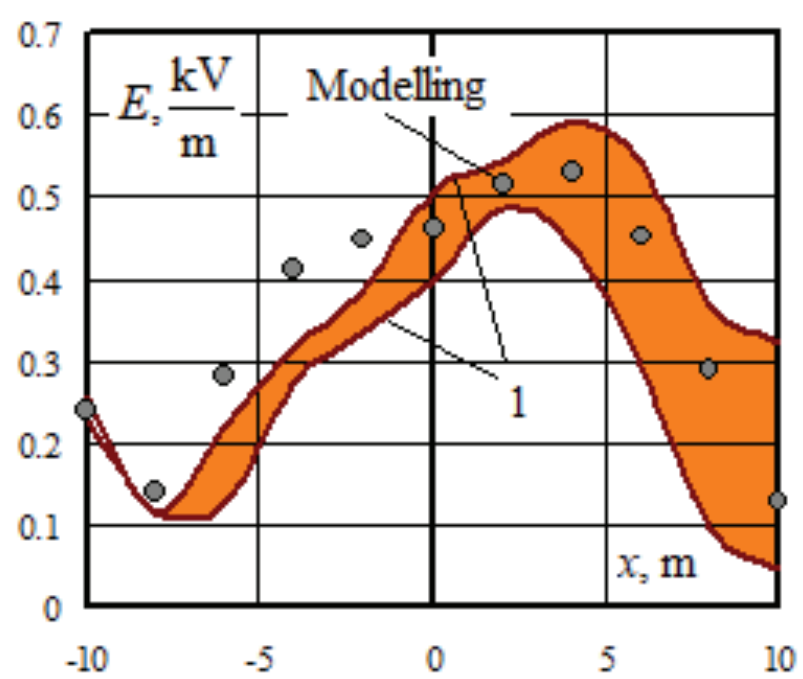

a)

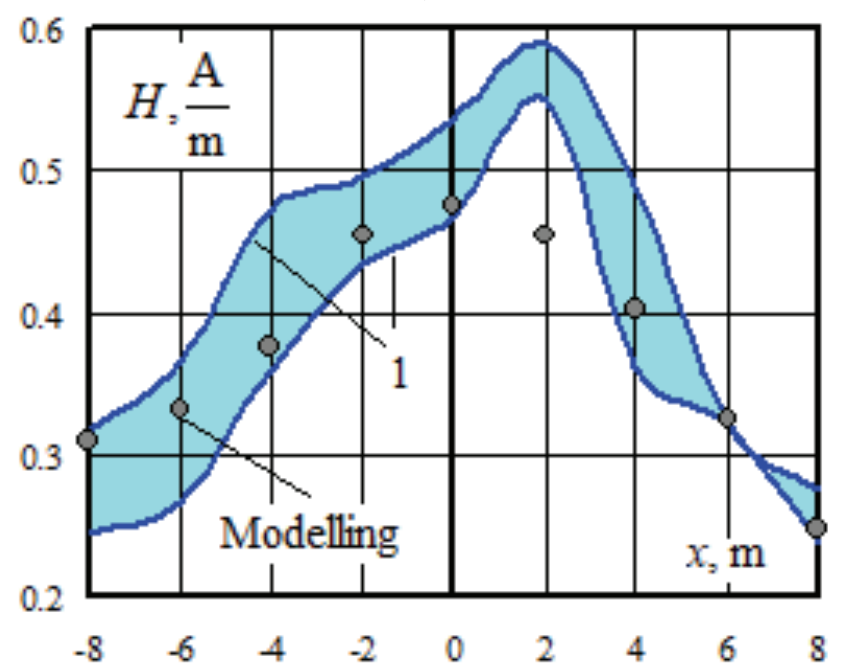

b)

Fig. 5. Interval representation of the EMF intensity measurement results: $a$-electric field; $b$ - magnetic field; 1 boundaries of scatter area of the experimental data.

Measurements were carried out with a P3-50 field intensity meter using projections of intensities on corresponding coordinate axes at a height of $1.5 \mathrm{~m}$ from the ground surface.

The results of measurements and computations are presented in Fig. 5.

In general, the nature of the relationship between the electric field intensity and the $\mathrm{x}$ coordinate, obtained by computation, corresponds to similar relationships constructed based on experiments. In the first experiment, the maximum intensity values are virtually equal to the calculated values.

The calculated values for the OPL right-hand circuit lie within the boundaries of the scatter area of the experimental values.

Adequacy of modeling is confirmed by high values of correlation coefficients between experimental and calculated data.

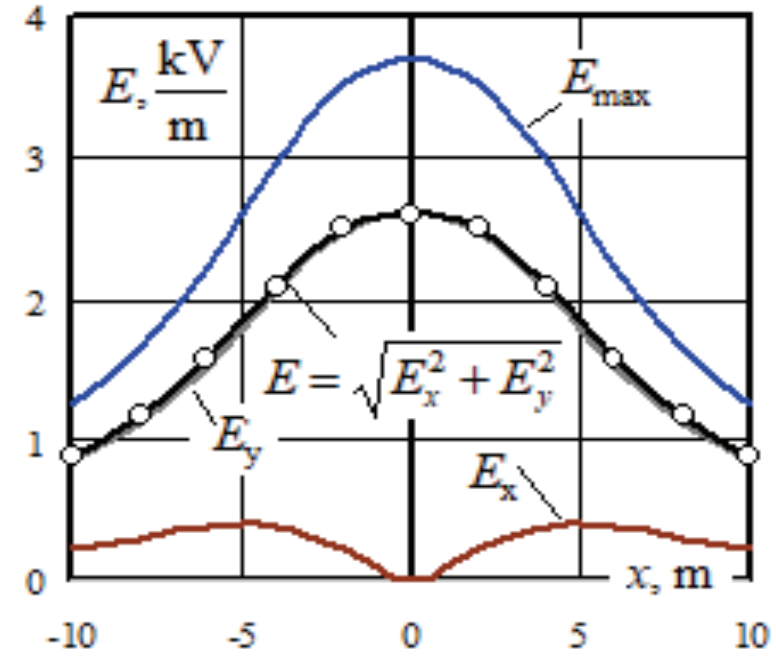

a)

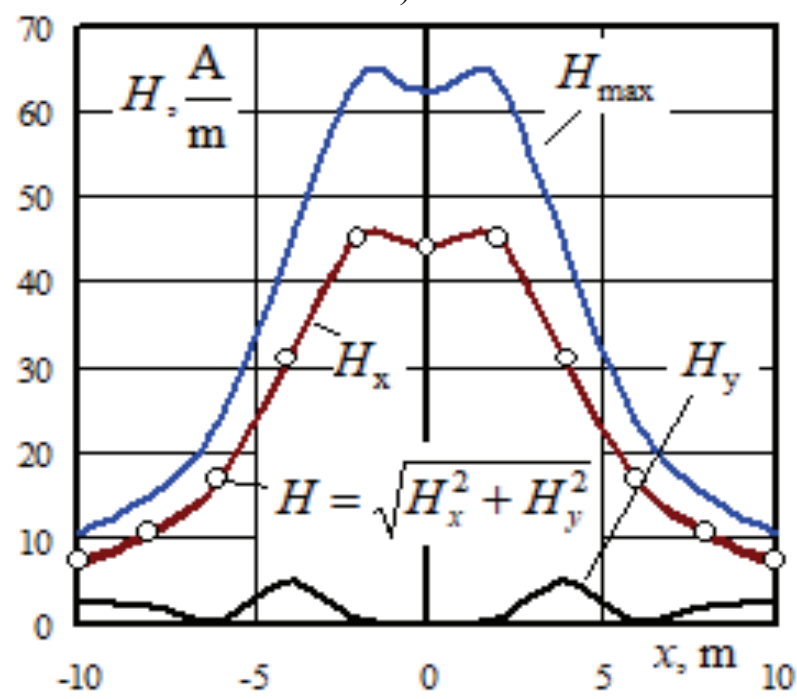

b)

Fig. 6. Amplitudes, effective values and components of EMF intensities at the height of $1.8 m$ : $a$-electric field; $b$-magnetic field.

\section{CASE STUdiES TO DETERMINE THE EMF INTENSITIES}

Figures 6-8 present the simulation results of EMF intensities at the height of $1.8 \mathrm{~m}$ for a typical $25 \mathrm{kV}$ TPSS of the double-track road section with a current of $414 \mathrm{~A}$ and voltage of $25 \mathrm{kV}$ of overhead catenaries.

The dependences obtained indicate the following:

- the low voltage of the traction network results in the maximum level of electric field intensity below the standard value of $5 \mathrm{kV} / \mathrm{m}$;

- the level of magnetic field intensity is quite close to the maximum permissible value but with the current of overhead catenaries equal to 414 A, EMF does not exceed it;

- electromagnetic energy density reaches $80 \mathrm{kV}-\mathrm{A} / \mathrm{m}^{2}$ near the center of the traction network. These data may serve as a basis for specifying electromagnetic safety standards in AC traction networks. 


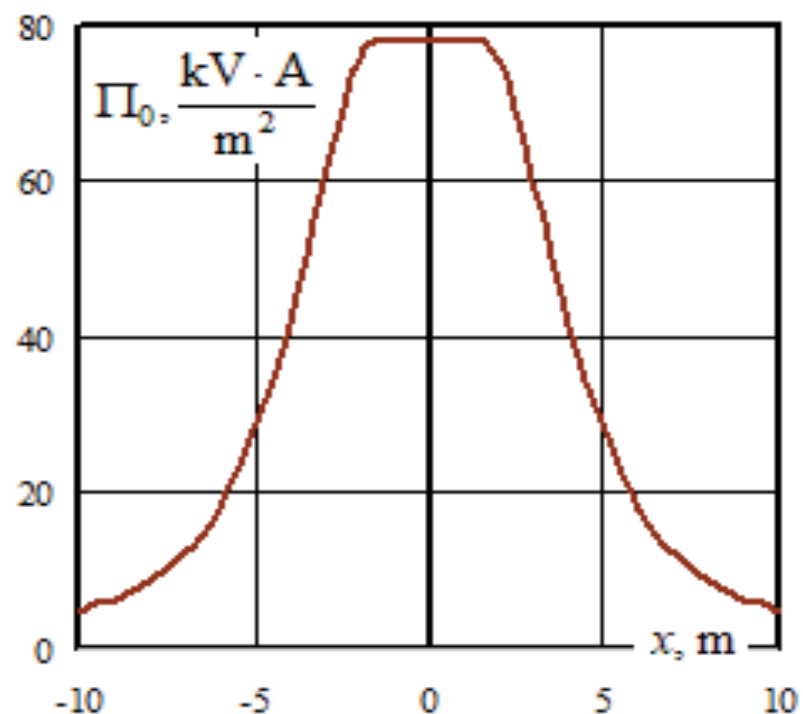

Fig. 7. Density of electromagnetic energy flux at the height of $1.8 \mathrm{~m}$.

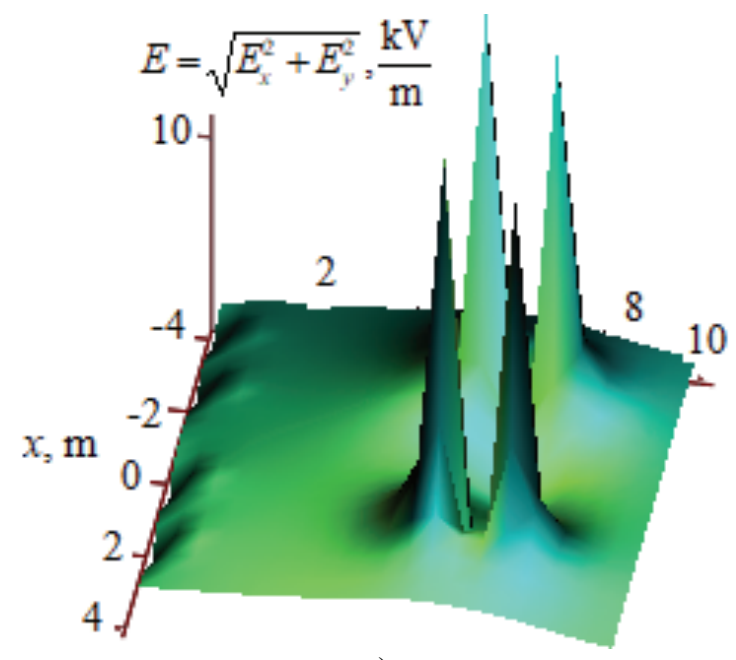

a)

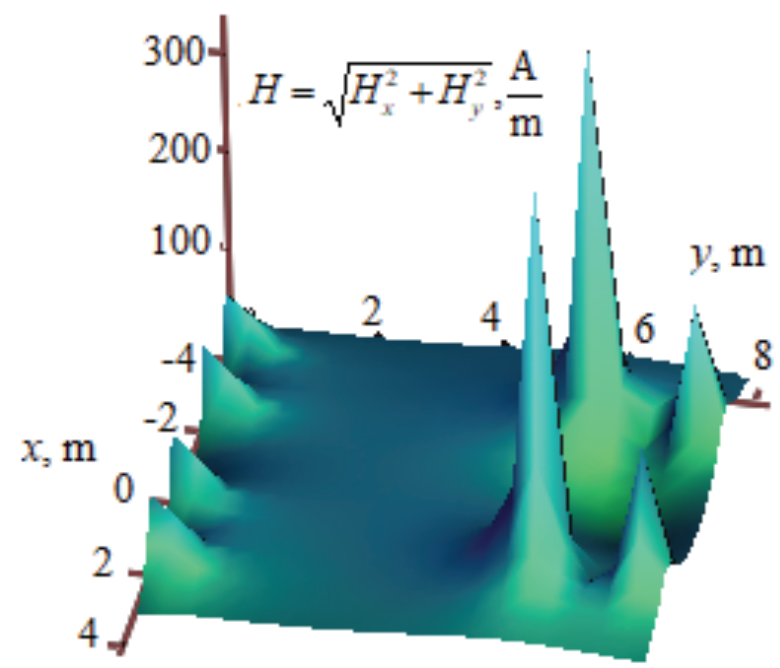

b)

Fig. 8. Cross-section of EMF intensity surfaces: $a$-electric field; $b$ - magnetic field.

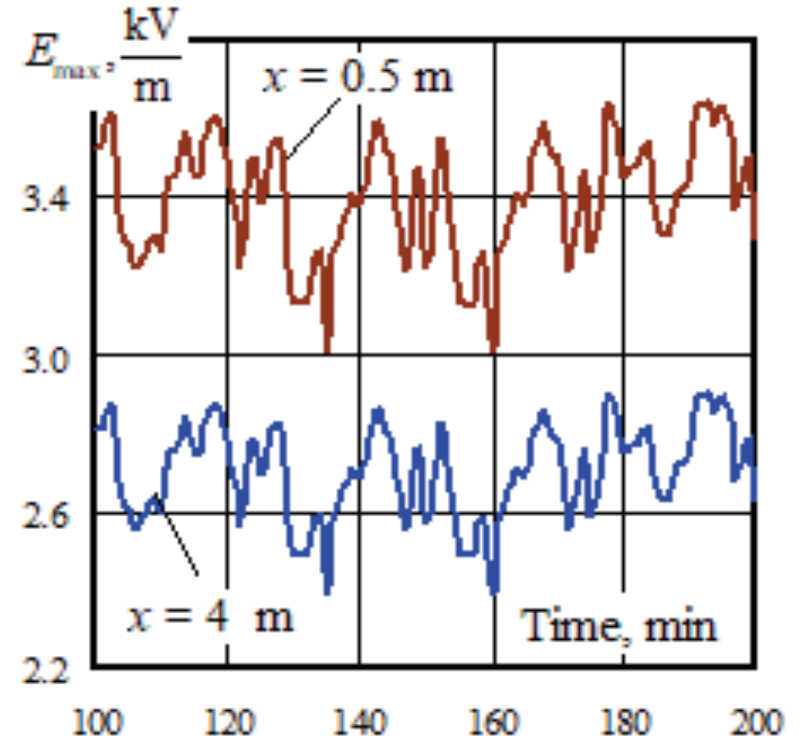

a)

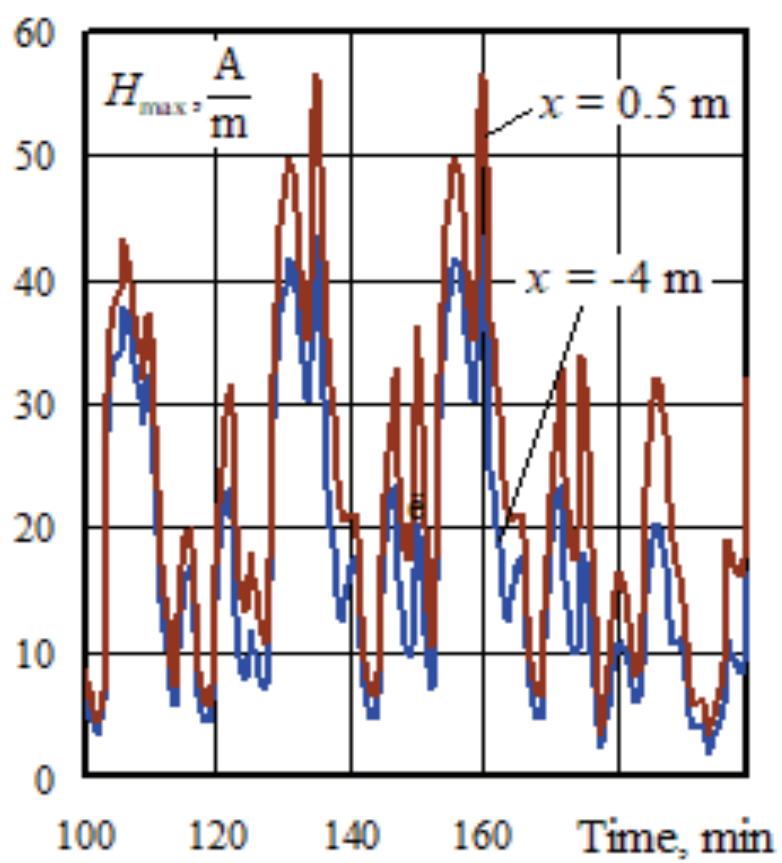

b)

Fig. 9. Time dependences of the intensity amplitude values: $a$-electric field; $b$ - magnetic field

Results of simulation of the EMF intensity changing over time at the height of $1.8 \mathrm{~m}$ are presented in Fig. 9.

Figure 10 shows the simulation results for the electromagnetic fields generated by a double-circuit 220 $\mathrm{kV}$ line with a current of $300 \mathrm{~A}$.

The presented results confirm the applicability of the considered technique for determining EMF when assessing the conditions of electromagnetic safety in traction networks and on the routes of high-voltage OPL. It is worth noting that this technique can be used in the EMF calculations for overhead power lines, cable power lines, and conductors of various designs. 


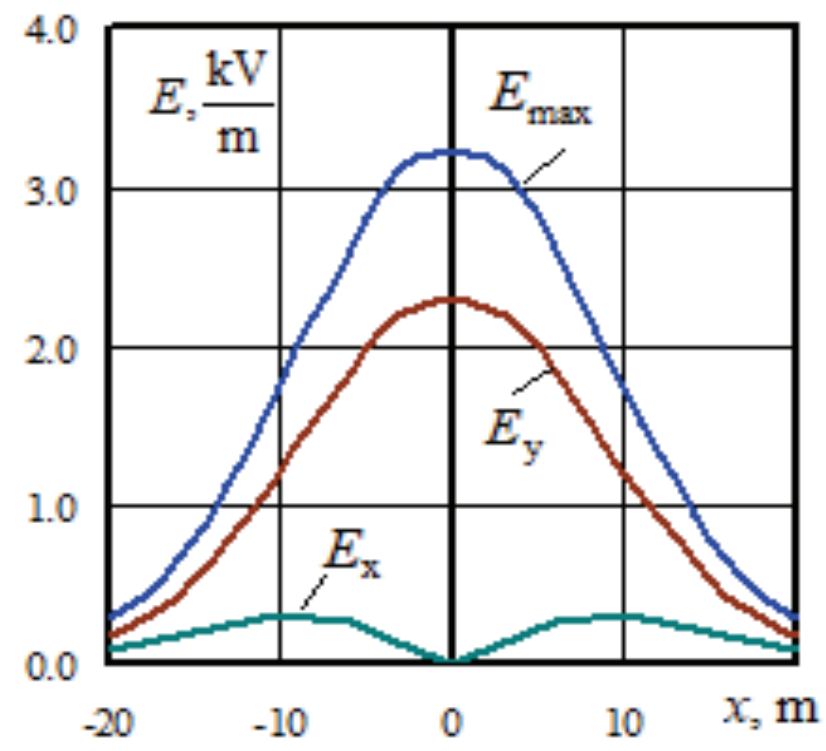

a)

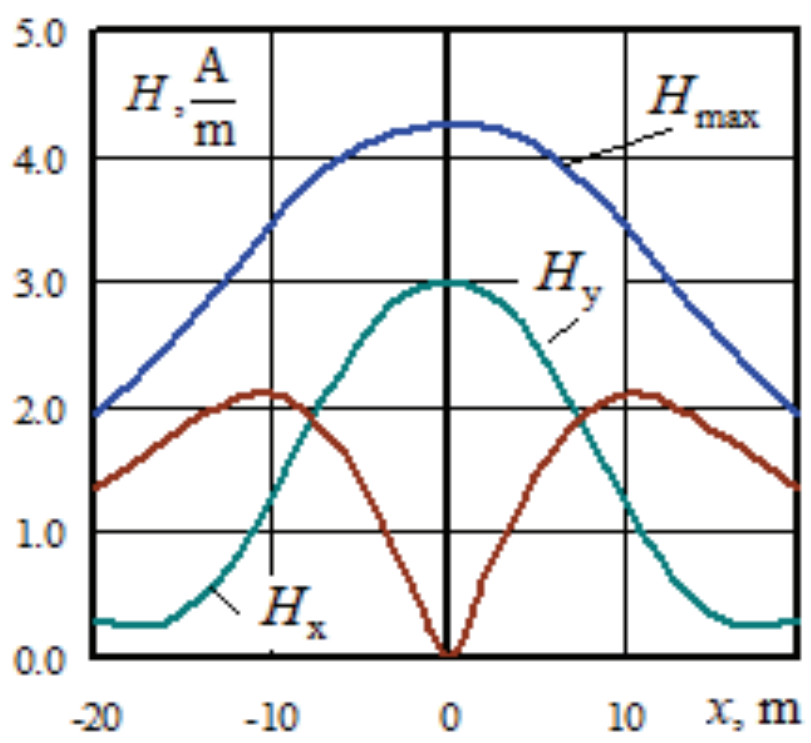

b)

Fig. 10. The intensity components versus the $x$-coordinate: $a$-electric field; $b$ - magnetic field

\section{EMF AT THE INTERSECTIONS OF TRACTION NETWORKS AND HIGH-VOLTAGE POWER LINES}

The above-described technique was extended to the detailed analysis of the electromagnetic field structure at points of power lines crossing the railroad.

Assuming mutual perpendicularity of the overhead power line and railroad wires, we can calculate components of EMF vectors and determine the resultant field, which is complicated by the following factors:

- overhead power line can be either single-circuit or double-circuit;

- mutual voltage phasing of TPSS and OPL, the spatial location of the OPL wires of different phases, and the number of electrified railroad tracks are to be factored in.
The problem statement at issue takes into account both these factors. Fig. 11 shows a frequently encountered situation where a $220 \mathrm{kV}$ line is crossing a double-track road section. In this Figure, the overhead catenary wires and rails of the odd track are given in black, while those of the even track are in gray. The dotted line shows OPL wires. Different systems of coordinates are chosen for railroad and OPL, which simplifies the calculation of fields. The coordinates are determined for the conditions where the $Z$-axis is directed along the railroad and the $Z_{\mathrm{T}}$ axis goes along the OPL wires. Currents have opposite directions to $Z$ and $Z_{\mathrm{T}}$ axes directions. The origins of coordinates are located on the railroad axis. In this case, electromagnetic fields can be calculated with the Fazonord software separately for traction network and overhead power line with the subsequent addition of respective field vector components.

In the $X Y Z$ coordinate system of railroad, the intensities of traction network electromagnetic field are calculated using formulas (3). In the $X_{\mathrm{T}} Y_{\mathrm{T}} Z_{\mathrm{T}}$ coordinate system of overhead power line, similar relations are written as follows:

$$
\begin{gathered}
\dot{E}_{\mathrm{T} X}=\frac{2}{\pi \varepsilon_{0}} \sum_{i=1}^{N_{\mathrm{T}}} \dot{\tau}_{i} \frac{\left(x_{\mathrm{T}}-x_{\mathrm{T} i}\right) y_{\mathrm{T}} y_{\mathrm{T} i}}{\lambda_{\mathrm{T} i}} ; \\
\dot{E}_{\mathrm{T} Y}=-\frac{1}{\pi \varepsilon_{0}} \sum_{i=1}^{N_{\mathrm{T}}} \dot{\tau}_{i} \frac{y_{\mathrm{T} i}\left[\left(x_{\mathrm{T}}-x_{\mathrm{T} i}\right)^{2}-y_{\mathrm{T}}^{2}+y_{\mathrm{T} i}^{2}\right]}{\lambda_{\mathrm{T} i}} ; \\
\dot{H}_{\mathrm{T} X}=\frac{1}{2 \pi} \sum_{i=1}^{N_{\mathrm{T}}} \dot{I}_{i} \frac{y_{\mathrm{T}}-y_{\mathrm{T} i}}{\xi_{\mathrm{T} i}} ; \dot{H}_{\mathrm{T} Y}=-\frac{1}{2 \pi} \sum_{i=1}^{N_{\mathrm{T}}} \dot{I}_{i} \frac{x_{\mathrm{T}}-x_{\mathrm{T} i}}{\xi_{\mathrm{T} i}} ; \\
\dot{E}_{\mathrm{T} Z}=0 ; \dot{H}_{\mathrm{TZ}}=0,
\end{gathered}
$$

where

$$
\begin{gathered}
\xi_{\mathrm{T} i}=\left[\left(x_{\mathrm{T}}-x_{\mathrm{T} i}\right)^{2}+\left[\left(y_{\mathrm{T}}+y_{\mathrm{T} i}\right)^{2} \mathrm{C}\right]\left[\left(x_{\mathrm{T}}-x_{\mathrm{T} i}\right)^{2}+\left[\left(y_{\mathrm{T}}-y_{\mathrm{T} i}\right)^{2}\right] ;\right.\right. \\
\lambda_{\mathrm{T} i}=\left(x_{\mathrm{T} i}-x_{\mathrm{T}}\right)^{2}+\left[\left(y_{\mathrm{T} i}-y_{\mathrm{T}}\right)^{2} .\right.
\end{gathered}
$$

In the formulas, $N$ is the number of traction network wires, $N_{T}$ is the number of OPL wires; $\dot{\tau}_{i}$ is charge per unit of length of wire $i, \dot{I}_{i}$ is current of wire $i$ in the direction opposite to the direction of $Z$ or $Z_{\mathrm{T}}$ axis; $\left(x_{i}, y_{i}\right),\left(x_{\mathrm{T} i}, y_{\mathrm{T} i}\right)$ are wire coordinates in respective cross-sections.

Observation point coordinates in the $X_{\mathrm{T}} Y_{\mathrm{T}} Z_{\mathrm{T}}$ system are related to the $X Y Z$ coordinates with following relationships on an assumption that origins of coordinates concur at one point:

$$
x=z_{\mathrm{T}} ; y=y_{\mathrm{T}} ; z=-x_{\mathrm{T}} .
$$

The components of OPL field vectors are similarly transformed from the $X_{\mathrm{T}} Y_{\mathrm{T}} Z_{\mathrm{T}}$ coordinate system to the $X Y Z$ system:

$$
\begin{aligned}
& \dot{E}_{X}^{\prime}=\dot{E}_{\mathrm{T} Z} ; \dot{E}_{Y}^{\prime}=\dot{E}_{\mathrm{TY}} ; \dot{E}_{Z}^{\prime}=-\dot{E}_{\mathrm{T} X} ; \\
& \dot{H}_{X}^{\prime}=\dot{H}_{\mathrm{T} Z} ; \dot{H}_{Y}^{\prime}=\dot{H}_{\mathrm{T} Y} ; \dot{H}_{Z}^{\prime}=-\dot{H}_{\mathrm{T} X} .
\end{aligned}
$$

The operations necessary to calculate field intensities at a specified point with coordinates $(x, y, z)$ are as follows:

1. Determine the components of intensities of the field created by railroad traction network by the given coordinates $(x, y, z)$ with the aid of Fazonord software in 


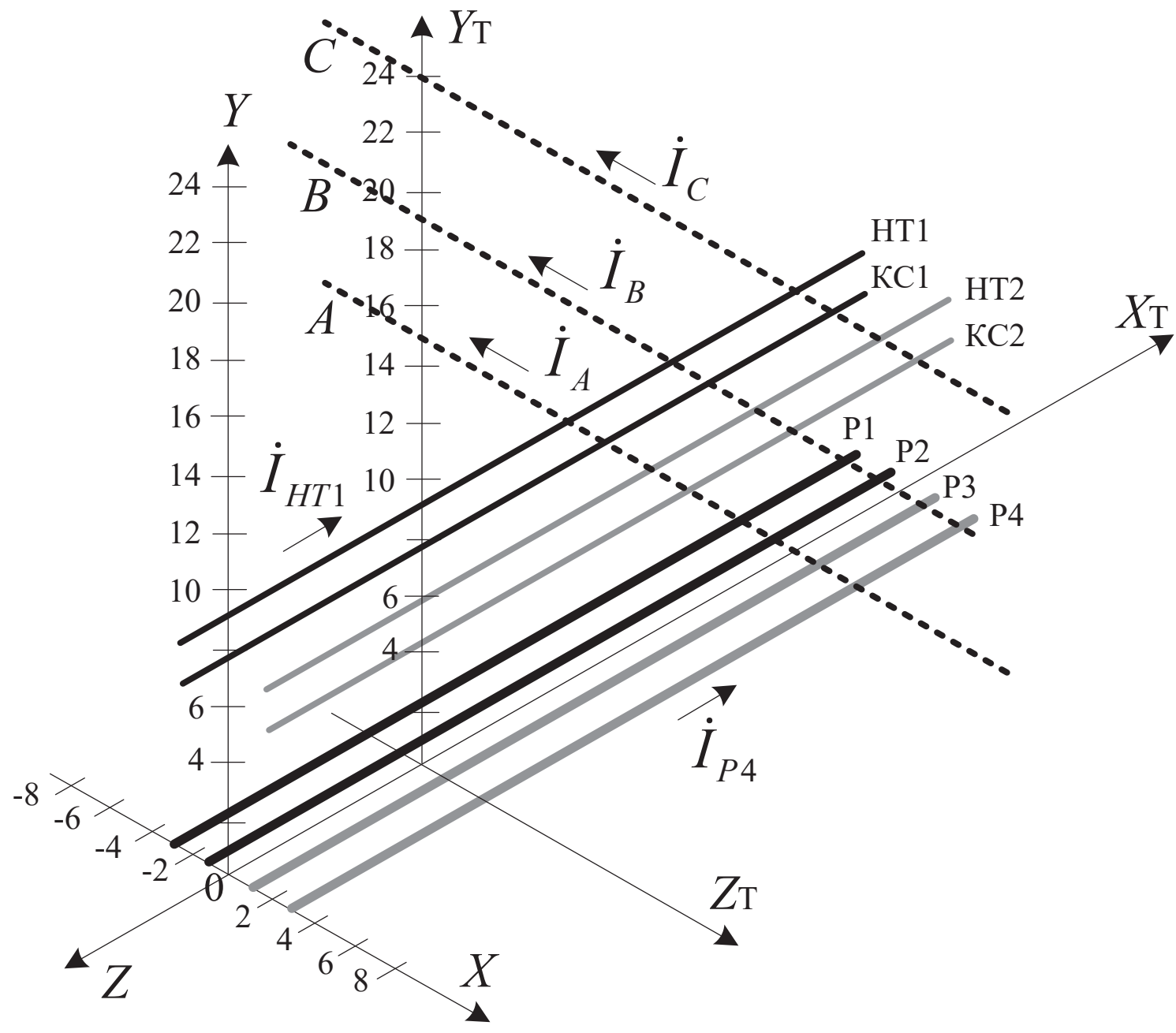

Fig. 11. The diagram of mutual location of OPL and traction network.

which the electromagnetic field intensity is determined after the operating conditions are calculated.

2. Determine the components of intensities of the field generated by overhead power line by the specified coordinates $z_{\mathrm{T}}=x ; y_{\mathrm{T}}=y ; x_{\mathrm{T}}=-z$ using the Fazonord software.

3. Calculate the total components of intensities at a specified point according to the expressions (4):

$$
\begin{gathered}
\dot{E}_{\Sigma X}=\dot{E}_{X}+\dot{E}_{\mathrm{T} Z}=\dot{E}_{X} ; \dot{E}_{\Sigma Y}=\dot{E}_{Y}+\dot{E}_{\mathrm{T} Y} ; \\
\dot{E}_{\Sigma Z}=\dot{E}_{Z}-\dot{E}_{\mathrm{T} X}=-\dot{E}_{\mathrm{T} X} ; \\
\dot{H}_{\Sigma X}=\dot{H}_{X}+\dot{H}_{\mathrm{T} Z}=\dot{H}_{X} ; \dot{H}_{\Sigma Y}=\dot{H}_{Y}+\dot{H}_{\mathrm{T} Y} ; \\
\dot{H}_{\Sigma Z}=\dot{H}_{Z}-\dot{H}_{\mathrm{T} X}=-\dot{H}_{\mathrm{T} X} .
\end{gathered}
$$

Thus, we determine all three components of the intensity vectors of electric and magnetic fields:

$$
\begin{gathered}
\dot{E}_{\Sigma i}=E_{\Sigma i} e^{j \varphi_{i}} ; E_{\Sigma i}(t)=\sqrt{2} E_{\Sigma i} \sin \left(\omega t+\varphi_{i}\right) ; \\
\dot{H}_{\Sigma i}=H_{\Sigma i} e^{j \psi_{i}} ; H_{\Sigma i}(t)=\sqrt{2} H_{\Sigma i} \sin \left(\omega t+\psi_{i}\right) ; \\
i=x, y, z .
\end{gathered}
$$

Ends of vectors of electric and magnetic field intensities calculated at some point trace out ellipses lying in the plane determined by components of these vectors [21]. Whether the vector belongs to a specific plane can be shown by the product of two vectors of the field at different time instants. Vector of this product is perpendicular to the direction of the plane in which the terms lie. In particular, one can analyze two vectors at time $t=0$ and at current time $t$. The components of vector product $\vec{E}_{0} \times \vec{E}_{t}$ are determined as follows:

$$
\begin{aligned}
& \left(\vec{E}_{0} \times \vec{E}_{t}\right)_{X}=2 E_{\Sigma Y} E_{\Sigma Z} \sin \left(\varphi_{Y}-\varphi_{Z}\right) \sin (\omega t) ; \\
& \left(\vec{E}_{0} \times \vec{E}_{t}\right)_{Y}=2 E_{\Sigma X} E_{\Sigma Z} \sin \left(\varphi_{Z}-\varphi_{X}\right) \sin (\omega t) ; \\
& \left(\vec{E}_{0} \times \vec{E}_{t}\right)_{Z}=2 E_{\Sigma X} E_{\Sigma Y} \sin \left(\varphi_{X}-\varphi_{Y}\right) \sin (\omega t) .
\end{aligned}
$$

Squared absolute value of the vector product equals

$$
\left|\left(\vec{E}_{0} \times \vec{E}_{t}\right)\right|^{2}=\left(\vec{E}_{0} \times \vec{E}_{t}\right)_{X}{ }^{2}+\left(\vec{E}_{0} \times \vec{E}_{t}\right)_{Y}{ }^{2}+\left(\vec{E}_{0} \times \vec{E}_{t}\right)_{Z}{ }^{2} .
$$

These data show that ratios of the vector product components to the vector absolute value (vector direction cosines) do not depend on time, which indicates that intensity vector is located at any time instant in the same plane. Normal line to this plane is determined by a single vector with components equal to 


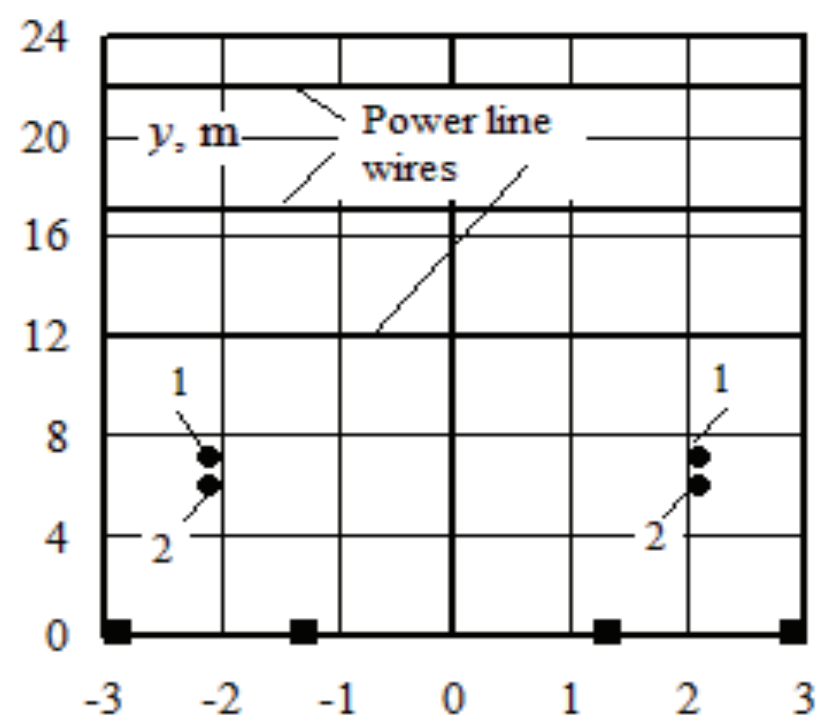

a)

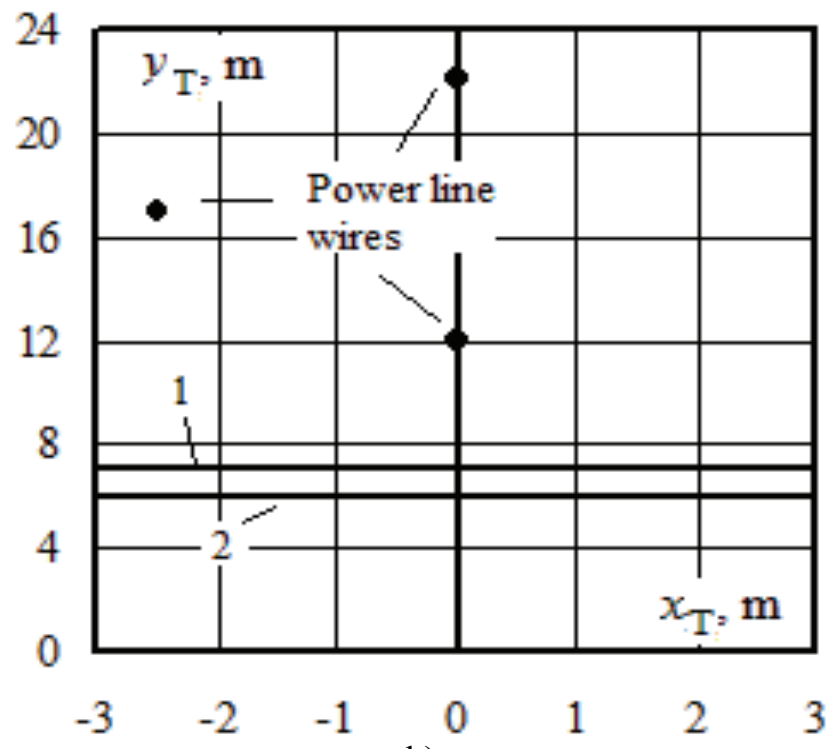

b)

Fig. 12. Location of traction network (a) and OPL (b) wires: 1-contact wire; 2 - catenary messenger.

$$
\frac{\left(\vec{E}_{0} \times \vec{E}_{t}\right)_{X}}{\left|\left(\vec{E}_{0} \times \vec{E}_{t}\right)\right|} ; \frac{\left(\vec{E}_{0} \times \vec{E}_{t}\right)_{Y}}{\left|\left(\vec{E}_{0} \times \vec{E}_{t}\right)\right|} ; \frac{\left(\vec{E}_{0} \times \vec{E}_{t}\right)_{Z}}{\left|\left(\vec{E}_{0} \times \vec{E}_{t}\right)\right|} .
$$

Extreme values of the intensity vector can be calculated by differentiating the squared vector length:

$$
\begin{aligned}
& E_{\mathrm{S}}=\left[E_{\Sigma i}(t)\right]^{2}=2 \sum_{i=1}^{3} E_{\Sigma i}^{2} \sin ^{2}\left(\omega t+\varphi_{i}\right)= \\
& =\sum_{i=1}^{3} E_{\Sigma i}^{2}-\sum_{i=1}^{3} E_{\Sigma i}^{2} \cos \left(2 \omega t+2 \varphi_{i}\right) .
\end{aligned}
$$

Extreme values are determined by zero value of the derivative:

$$
\frac{d E_{\mathrm{S}}}{d t}=2 \omega \sum_{i=1}^{3} E_{\Sigma i}^{2} \sin \left(2 \omega t+2 \varphi_{i}\right)=0,
$$

from which

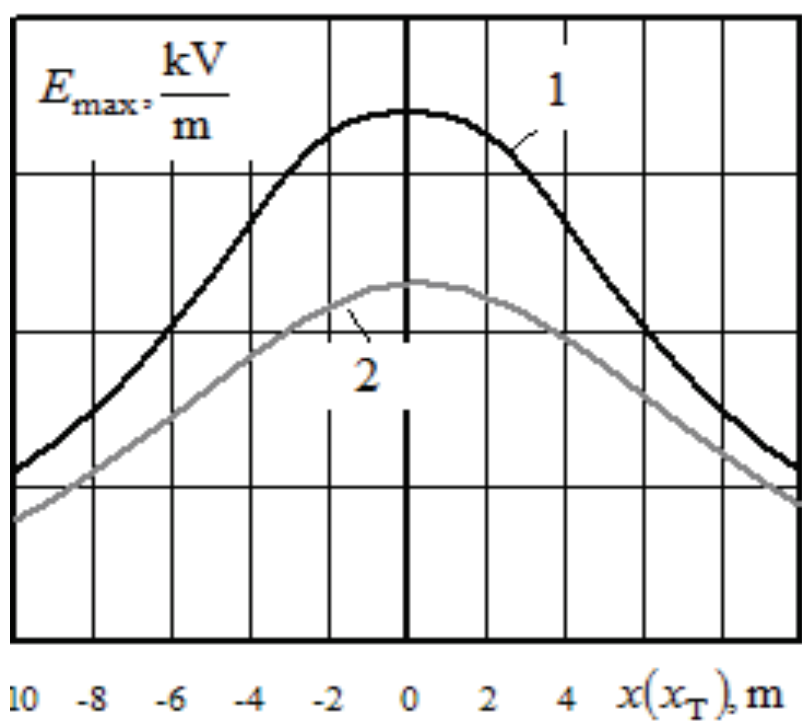

a)
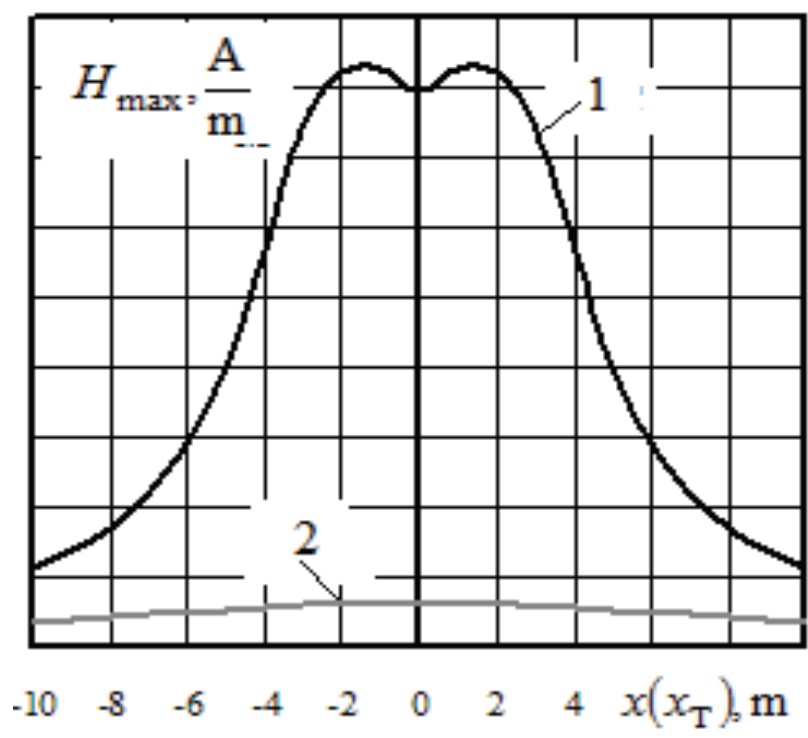

b)

Fig. 13. The amplitudes of electric (a) and magnetic (b) field intensities versus the $x$ coordinate: 1 -traction network; $2-O P L$.

$$
t_{\max , \min }=-\frac{1}{2 \omega} \operatorname{Arctg} \frac{\sum_{i=1}^{3} E_{\Sigma i}^{2} \sin \left(2 \varphi_{i}\right)}{\sum_{i=1}^{3} E_{\Sigma i}^{2} \cos \left(2 \varphi_{i}\right)},
$$

where the maximum is determined by negative value of the second derivative:

$$
\begin{aligned}
& \sin \left(2 \omega t_{\max }\right) \sum_{i=1}^{3} E_{\Sigma i}^{2} \sin \left(2 \varphi_{i}\right)> \\
& \cos \left(2 \omega t_{\max }\right) \sum_{i=1}^{3} E_{\Sigma i}^{2} \cos \left(2 \varphi_{i}\right),
\end{aligned}
$$

while the minimum is determined by positive value:

$$
\begin{aligned}
& \sin \left(2 \omega t_{\min }\right) \sum_{i=1}^{3} E_{\Sigma i}^{2} \sin \left(2 \varphi_{i}\right)< \\
& \cos \left(2 \omega t_{\min }\right) \sum_{i=1}^{3} E_{\Sigma i}^{2} \cos \left(2 \varphi_{i}\right) .
\end{aligned}
$$



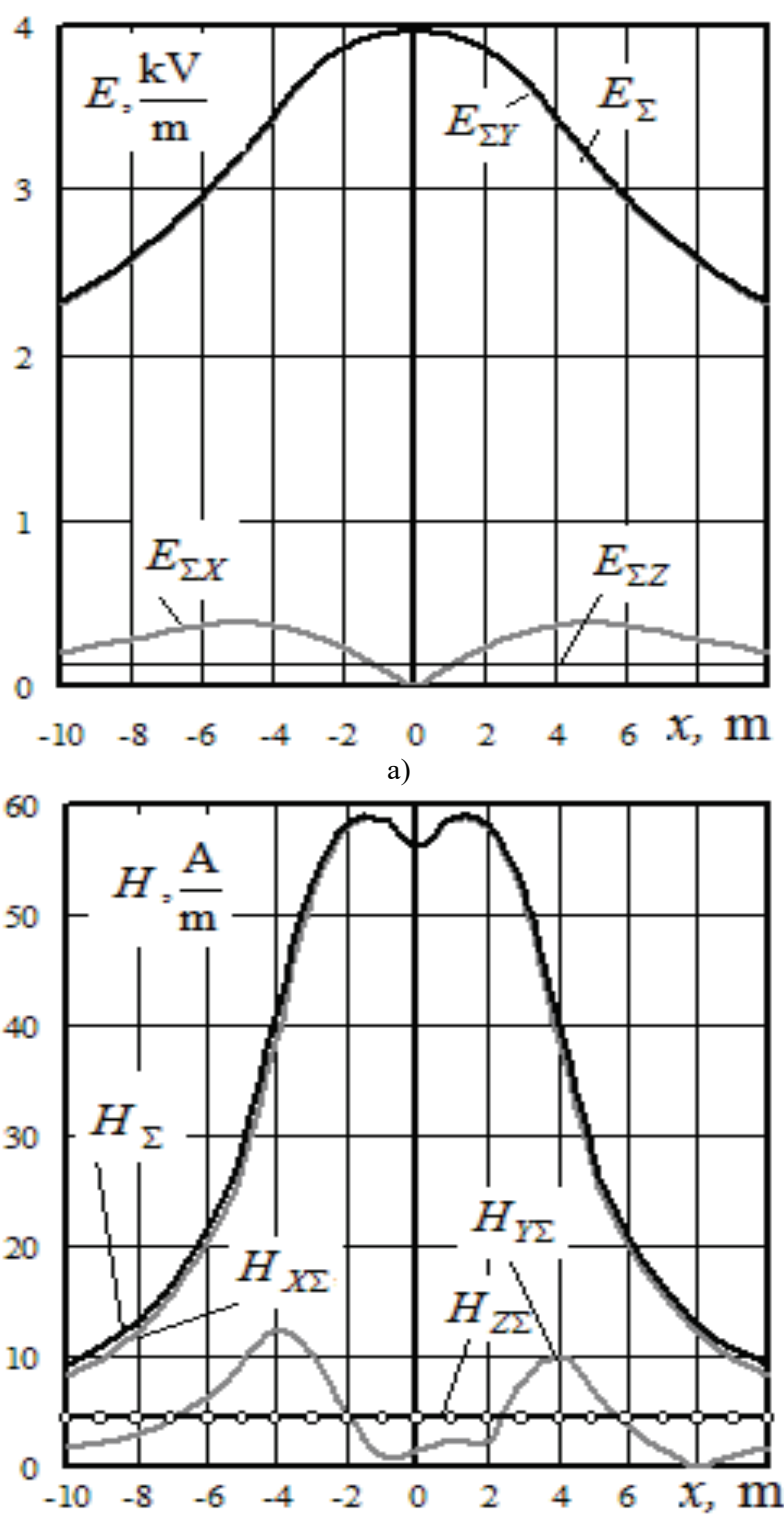

b)

Fig. 14. The total components of intensities for electric (a) and magnetic (b) fields (effective values) versus $x$ coordinate.

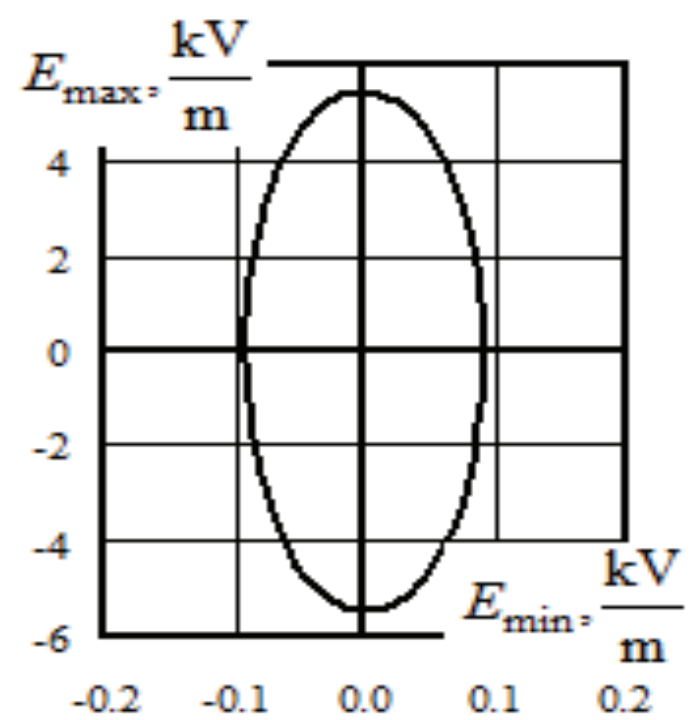

a)

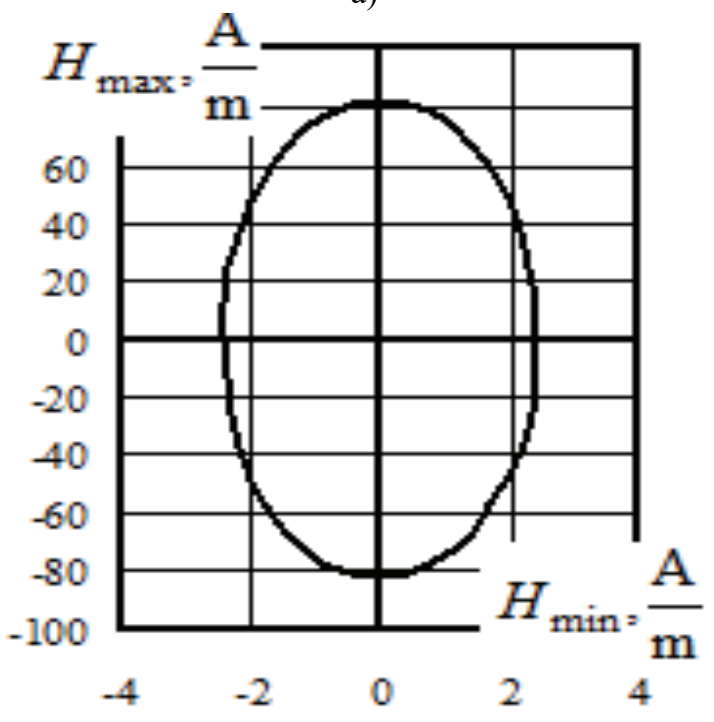

b)

Fig. 15. Loci of resultant intensity vectors of electric (a) and magnetic (b) fields in coordinate axes of polarization ellipse for the point with coordinates $x=2 m ; y=1.8 m$.

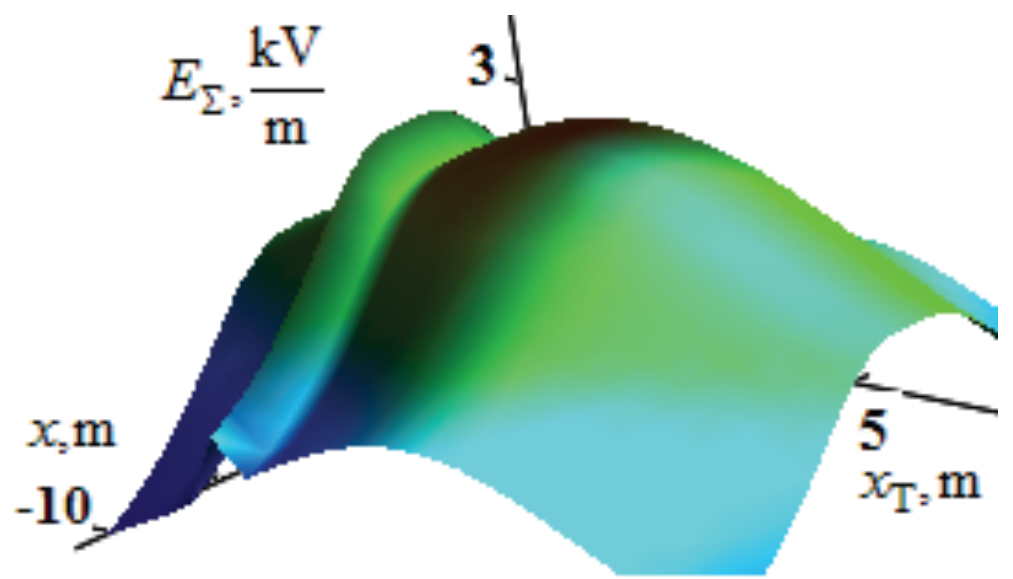

Fig. 16. The graph of the surface of electric field total intensity in $x, x T$ coordinates. 


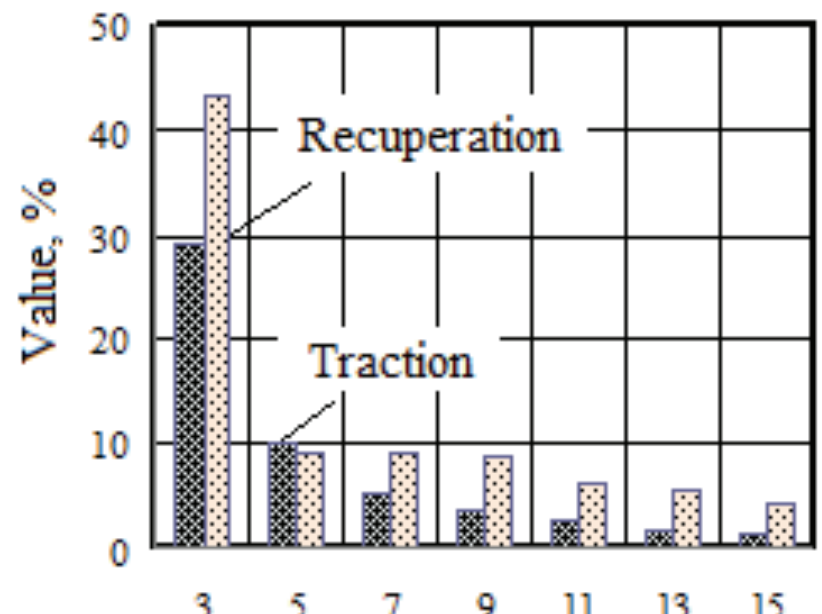

Fig. 17. Typical levels of electric locomotive current harmonics as a percentage of the fundamental frequency current.

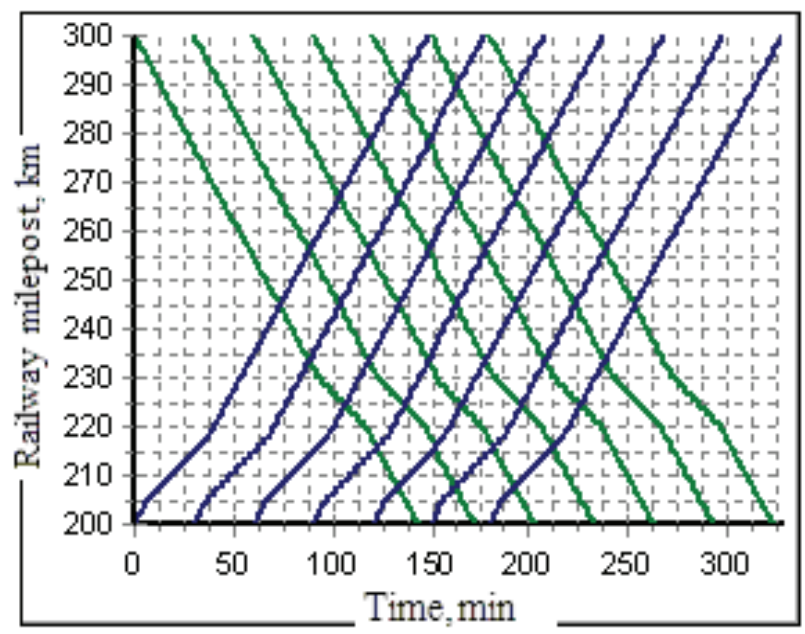

Fig. 19. Train schedule.

Different field points are characterized by different polarizations and field component relationships. Locus of the intensity vector located in the plane of polarization can be determined using the procedure from [4]. The simulation results of EMF of the wire systems according to Fig. 12 for the height of the observation point of $1.8 \mathrm{~m}$ are shown in Figs. 13-16.

Coordinate $z$ was measured from the point of intersection of the overhead power line (AC-300 wires) with the traction network. The overhead line was assumed to transit the power of $5+j 40 \mathrm{MV} \cdot \mathrm{A}$ by each phase with a linear voltage of $230 \mathrm{kV}$ and current of $648 \mathrm{~A}$, which are close to maximum permissible values for the AC-300 wires. Voltages at OPL starting end were equal to 132.8 $\mathrm{kV}$ with angles $0^{\circ},-120^{\circ}, 120^{\circ}$, currents flowing into these nodes were $648 \mathrm{e}^{-j 28.6^{\circ}} \mathrm{A}, 647 \mathrm{e}^{-j 148.6^{\circ}} \mathrm{A}$, and $647 \mathrm{e}^{j 91.3^{\circ}} \mathrm{A}$. The power of $8+j 8 \mathrm{MB} \cdot \mathrm{A}$ was transmitted by catenaries of each railway track. The calculated voltage of the overhead system was $25.6 \mathrm{e}^{-j 5.6^{\circ}} \mathrm{kV}$, and overhead catenaries currents were equal to $450 \mathrm{e}^{-j 51.1^{\circ}} \mathrm{A}$.

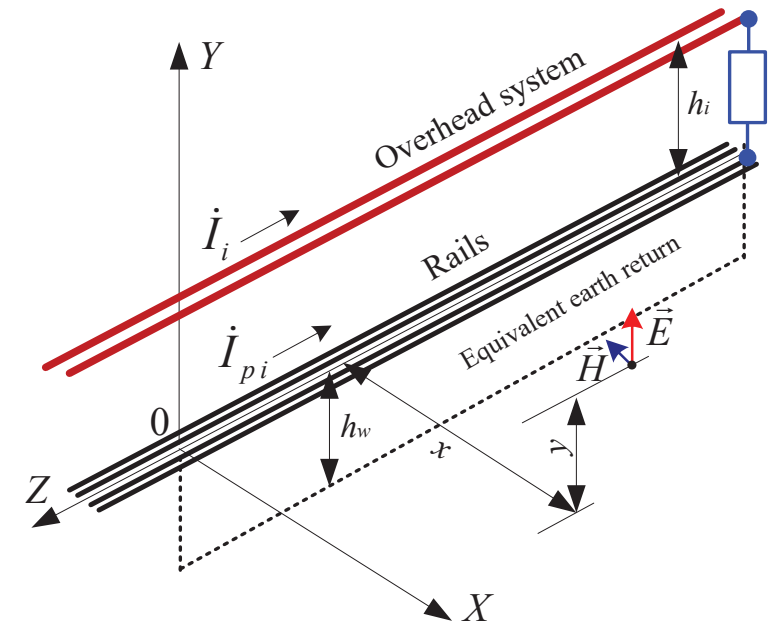

Fig. 18. Coordinate system exemplified by a traction network.

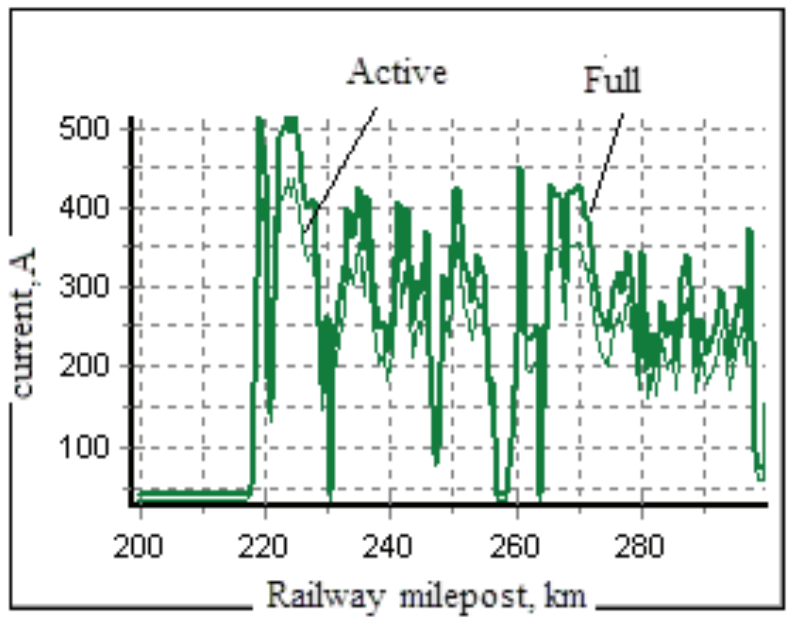

a)

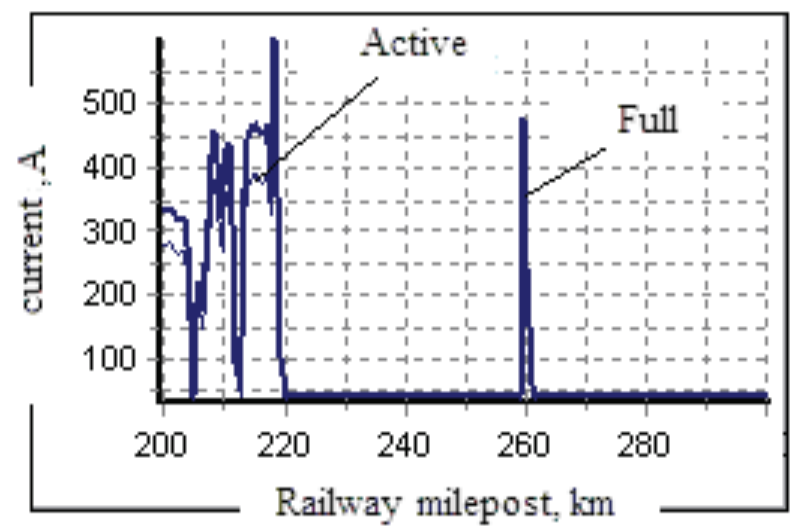

b)

Fig. 20. Train current profiles: $a$-odd direction; $b$ - even direction. 


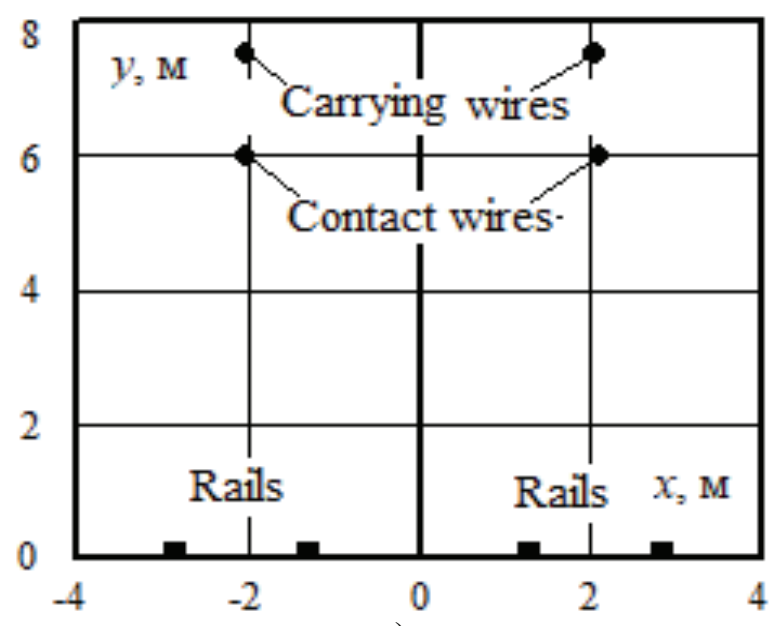

a)

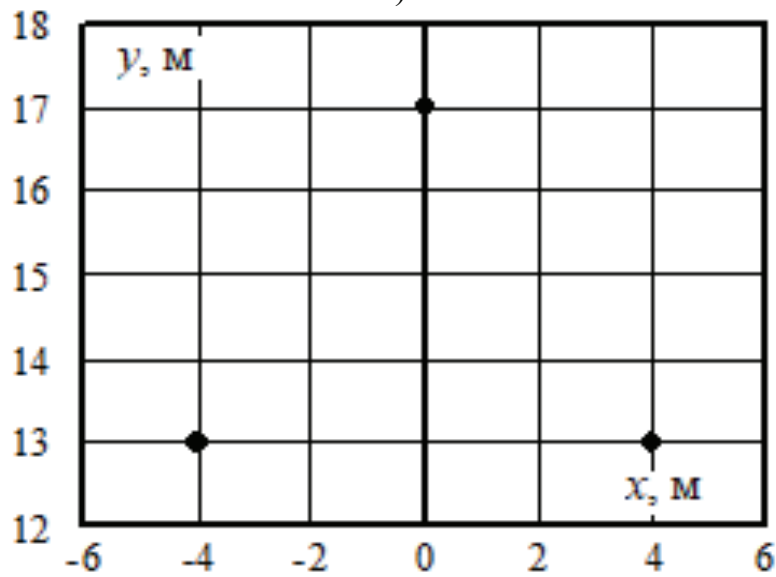

b)

Fig. 21. Coordinates of conductive parts: a-traction network; $b-O P L$.

The findings suggest the following.

1. The total intensities of magnetic fields of traction network and OPL differ from the intensity of magnetic field generated by traction network by $-2 \ldots+13 \%$. At the point with coordinates $x=2 \mathrm{~m} ; y=1.8 \mathrm{~m}$, the magnetic field intensity amplitude in the calculated option was equal to $82 \mathrm{~A} / \mathrm{m}$.

2. The increase in the maximum value of the electric field intensity amplitude at the intersection point of the OPL and traction network reaches $66 \%$; at the point indicated in the previous paragraph, the value of this parameter is $5.4 \mathrm{kV} / \mathrm{m}$.

The developed technique can be used to resolve practical issues associated with the improvement in electromagnetic safety for personnel engaged in the operation of high voltage power grids and AC railroad power supply systems.

\section{CONSIDERATION OF HIGHER HARMONICS IN EMF SIMULATION}

AC traction networks are the sources of higher harmonics, as their voltage often exceeds the standards. Russian rectifier electric locomotives with a rated voltage of $25 \mathrm{kV}$ not only consume the current from overhead catenary but also generate considerable higher
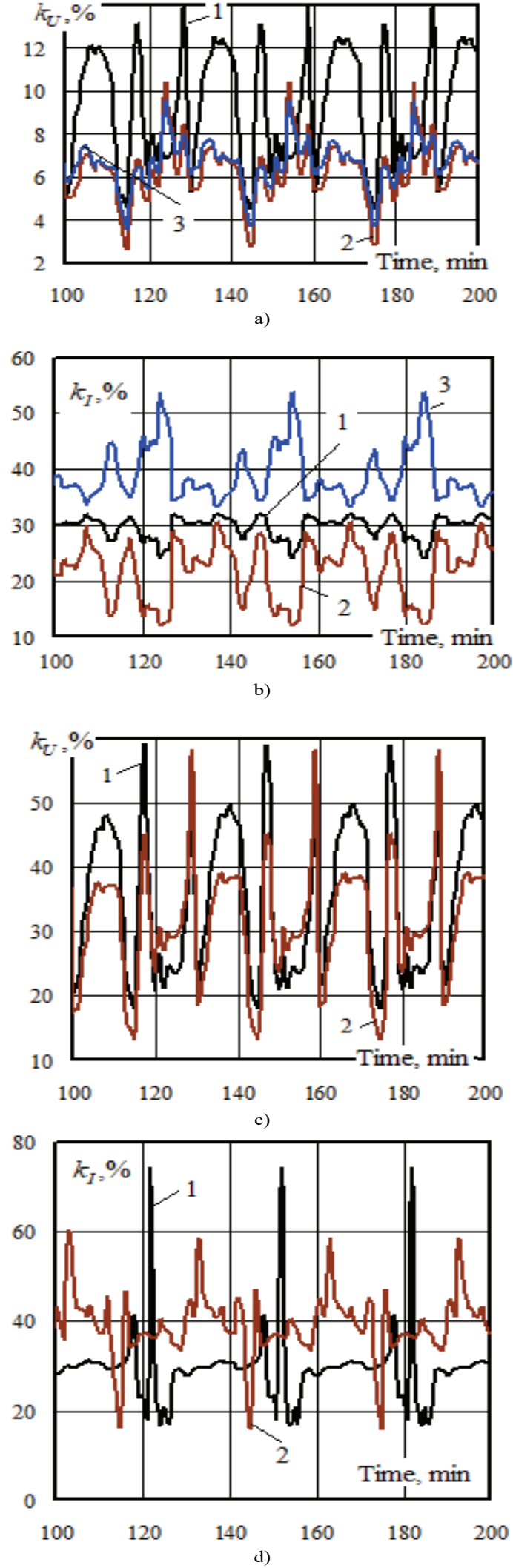

Fig. 22. The total harmonic current and voltage distortion factors versus simulation time: $a, b-O P L ; c, d$-overhead system; $k U, k I-t o t a l$ harmonic current and voltage distortion factors; in a, b: 1 -phase A; 2-phase B; 3 -phase $C$; in c, d: 1 -overhead catenary of the odd track; 2 -overhead catenary of the even track 

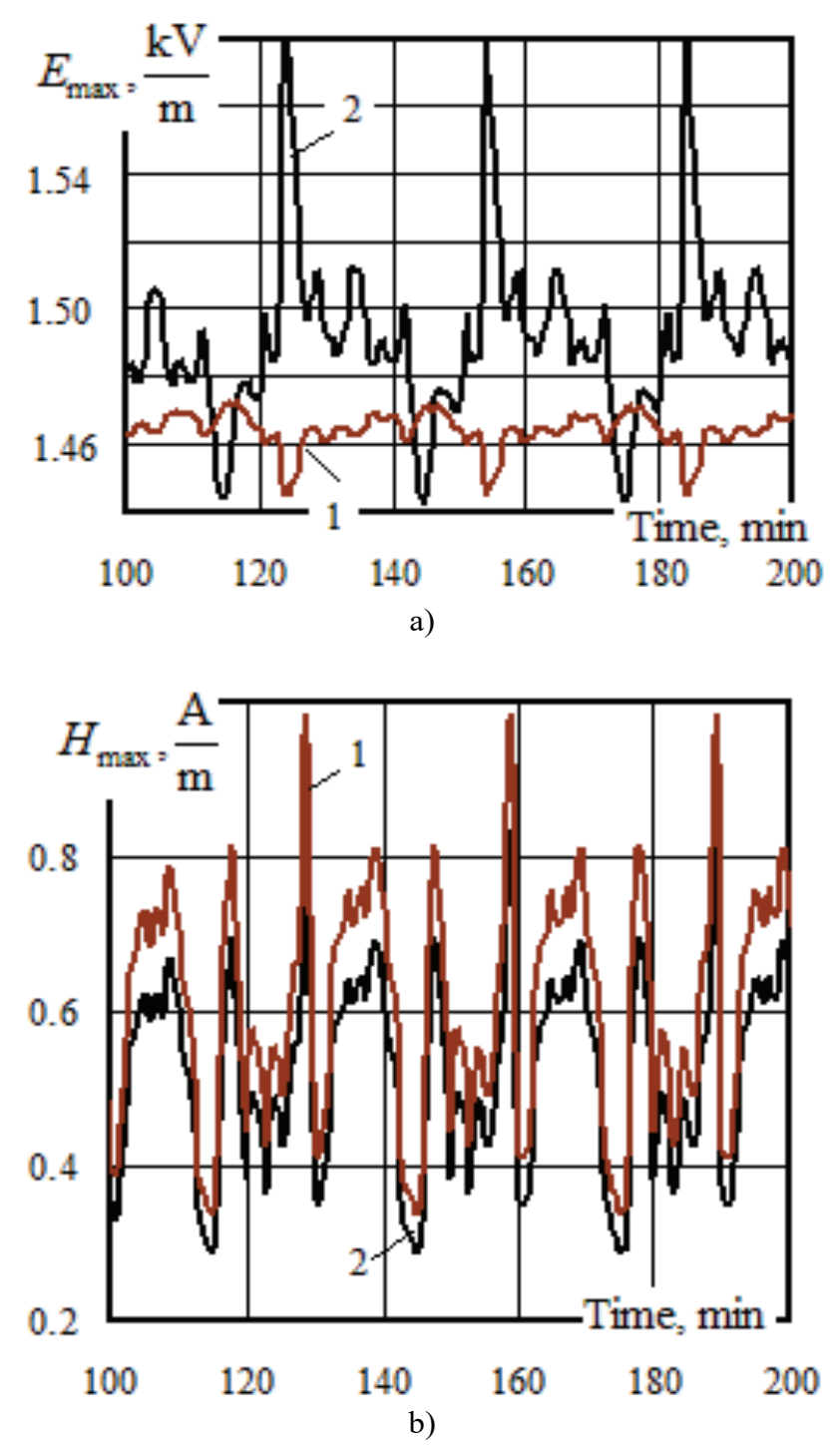

Fig. 23. Maximum intensities of EMF generated by OPL versus simulation time: $a$-electric field; $\sigma$-magnetic field; 1 -for fundamental frequency; 2 -for higher harmonics.

harmonics (Fig. 17), which entails significant distortions of current and voltage waveforms. Harmonics of traction networks penetrate into $110-220 \mathrm{kV}$ supply networks and increase harmonics of network voltage. For this reason, electromagnetic fields generated by a $25 \mathrm{kV}$ overhead system and 110-220 kV power lines supplying traction substations contain a significant proportion of higher harmonics [18]. This factor should be kept in mind when assessing the interference immunity of various types of equipment and when determining the electromagnetic safety conditions. Higher harmonics complicate the polarization picture [22-27], and the best way to simulate is to analyze the fields separately for different harmonics, with their subsequent superposition.

Calculation of EMF intensities requires a preliminary computation of operating conditions of single-phase traction network and external three-phase power supply
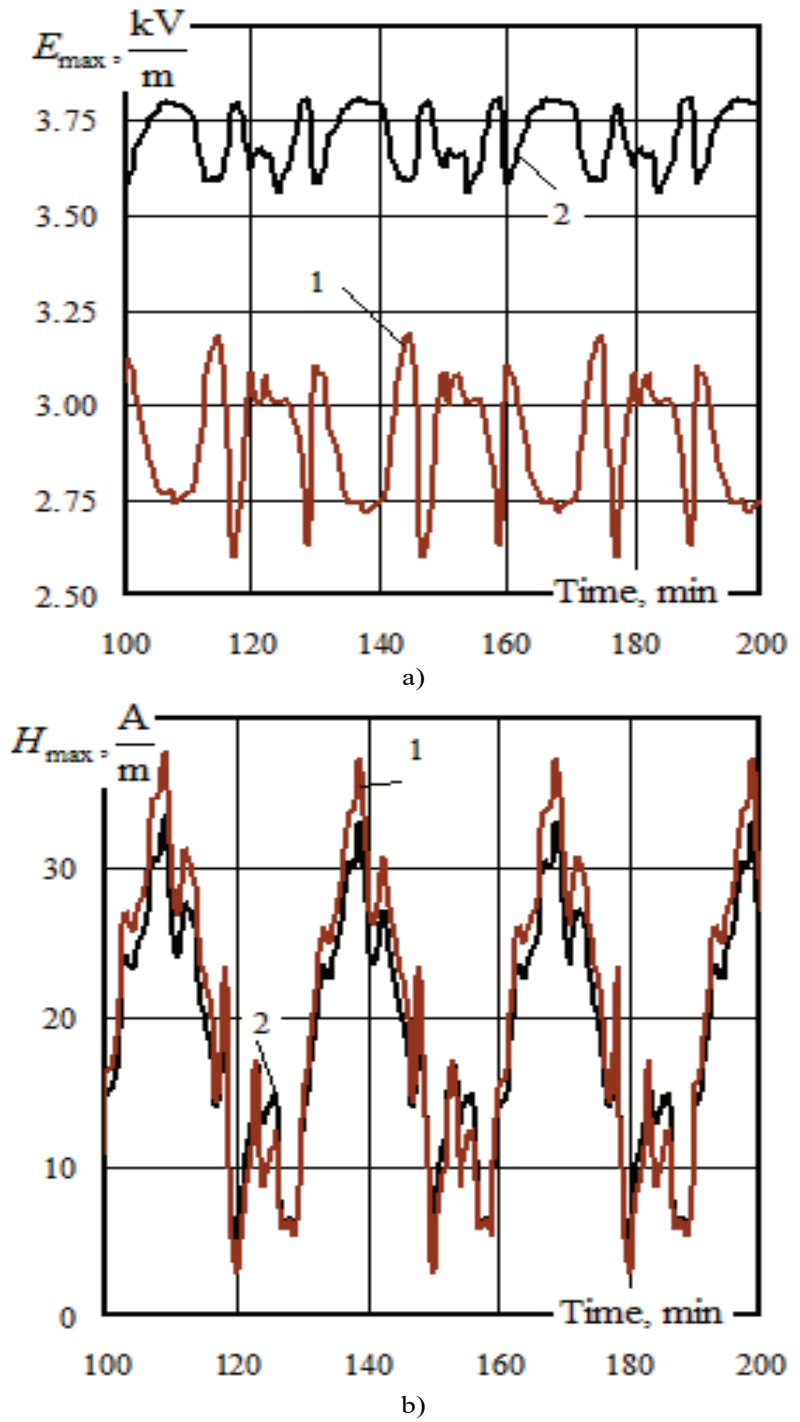

Fig. 24. Amplitudes of intensities of EMF generated by the traction network versus simulation time: $a$-electric field; $b-$ magnetic field; 1 -for fundamental frequency; 2 -for higher harmonics.

system. Nonstationarity and changes in the location of traction loads create additional difficulties in calculating the operating conditions, which are almost overcome for fundamental frequency in [18].

The changes in magnitudes and spatial location of traction loads are factored in by computations of a series of operating conditions satisfying the given time instants. The diagrams for such computations are usually built with the discreteness of 1 minute. The electromagnetic field of the traction network is determined by the voltage and current of the catenary wires and rails. Rail currents are calculated as induced currents of grounded conductors located on the ground surface. The electric and magnetic fields of parallel rectilinear wires are linearly polarized, the position of the intensity vectors changes during the period.

Electromagnetic fields of traction network and OPL are plane-parallel and identical in any plane perpendicular 


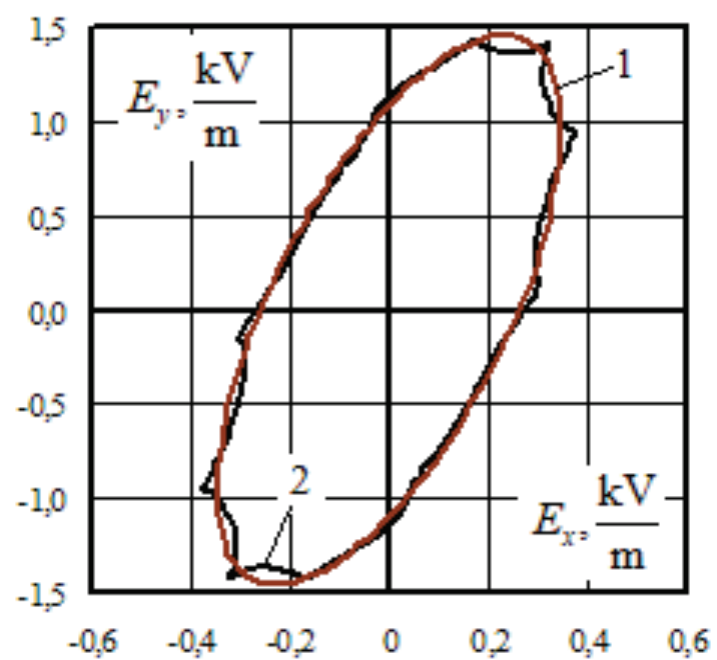

a)

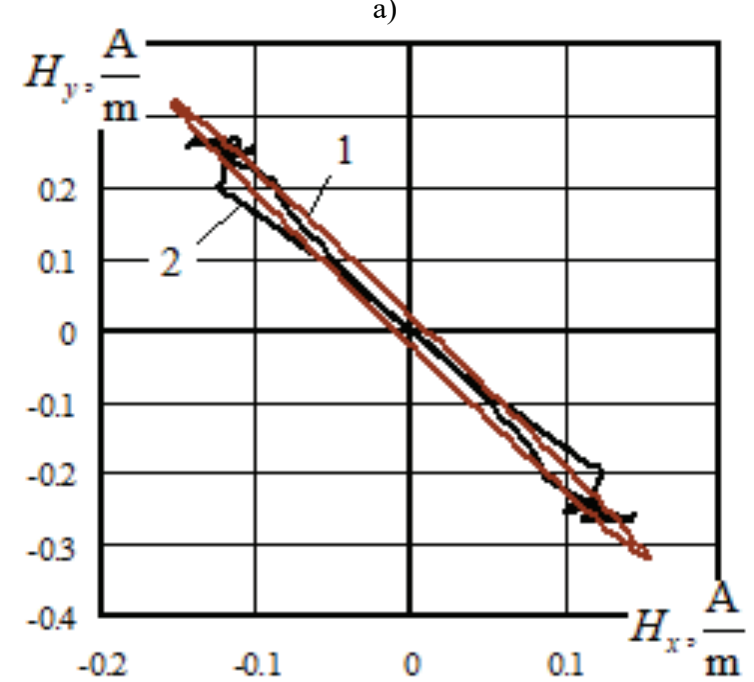

b)

Fig. 25. Loci of the OPL EMF intensity vectors at the 206th simulation minute: a-electric field; $b$-magnetic field; 1 -for fundamental frequency; 2 -for higher harmonics.

to the wires. As noted before, it is convenient to use the Cartesian coordinate system to describe such fields because the origin of the system lies on the ground surface under the center of the wire system, the $X$-axis is perpendicular to the wires along the ground surface, the $Y$-axis is directed vertically upward, and the $Z$-axis is directed against the positive direction of currents (Fig. 18).

Electric field intensities at a point with coordinates $x$, $y$ for each $k$ harmonic of the line, including $N$ wires, are calculated using the formulas similar to those provided above:

$$
\begin{gathered}
\dot{E}_{k x}=\frac{2}{\pi \varepsilon_{0}} \sum_{i=1}^{N} \dot{\tau}_{k i} \frac{\left(x-x_{i}\right) y y_{i}}{\xi_{i}} \\
\dot{E}_{k y}=-\frac{1}{\pi \varepsilon_{0}} \sum_{i=1}^{N} \dot{\tau}_{k i} \frac{y_{i}\left[\left(x-x_{i}\right)^{2}-y^{2}+y_{i}^{2}\right]}{\xi_{i}}
\end{gathered}
$$

where $\dot{\tau}_{k i}$ is the chargeofwire $i$ perunitoflength for harmonic $k$, determined from the first group of Maxwell's formulas

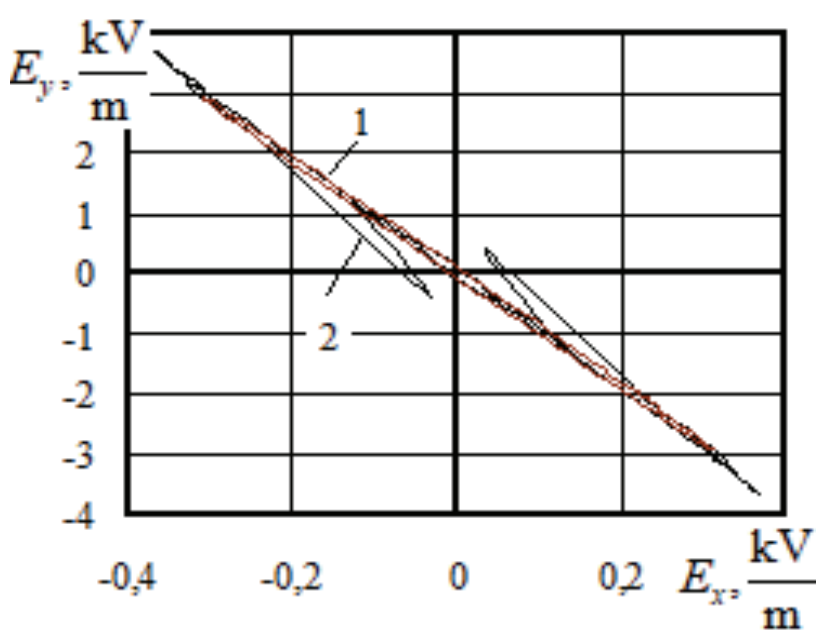

a)

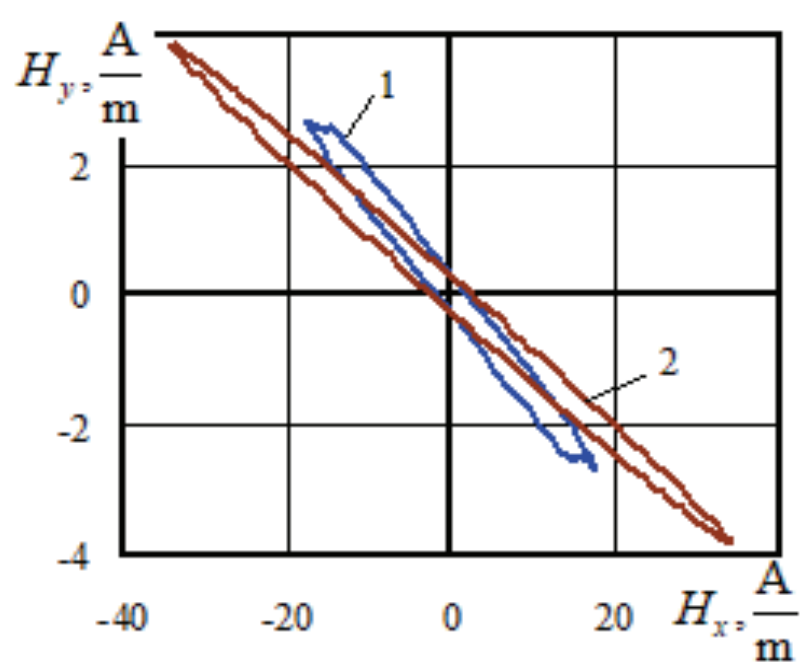

b)

Fig. 26. Loci of the traction network EMF intensity vectors at the 206th simulation minute: 1 - for fundamental frequency; 2 -for higher harmonics; $a$-electric field; $b$-magnetic field.

$\dot{\mathbf{T}}_{k}=\mathbf{A}^{-1} \cdot \dot{\mathbf{U}}_{k} ; \quad \dot{\mathbf{U}}_{k}=\left[\begin{array}{lll}\dot{U}_{k 1} & \ldots & \dot{U}_{k N}\end{array}\right]^{T}$ is the vector of wire potentials at harmonic $k ; \quad \dot{\mathbf{T}}_{k}=\left[\begin{array}{lll}\dot{\tau}_{k 1} & \ldots & \dot{\tau}_{k N}\end{array}\right]^{T}$ is the vector of linear charges of wires, $\mathbf{A}$ is the frequencyindependent matrix of potential coefficients.

For each electric field harmonic, the time dependences of spatial components are determined by the expression

$$
\vec{E}_{k}(t)=E_{m x} \sin \left(k \omega t+\psi_{k x}\right) \vec{e}_{x}+E_{m y} \sin \left(k \omega t+\psi_{k y}\right) \vec{e}_{y},
$$

where $\vec{e}_{x}, \vec{e}_{y}$ are unitary vectors of the Cartesian coordinate system; $\omega=314 \mathrm{rad} / \mathrm{s}$. As shown in [21], the vectors of field extreme values $\vec{E}_{k \max }$ and $\vec{E}_{k \min }$ are located in the polarization plane of a given harmonic and the intensity vector rotates in this plane with an ellipseshaped locus. The time instants satisfying the intensity extreme values are calculated for each harmonic based on 

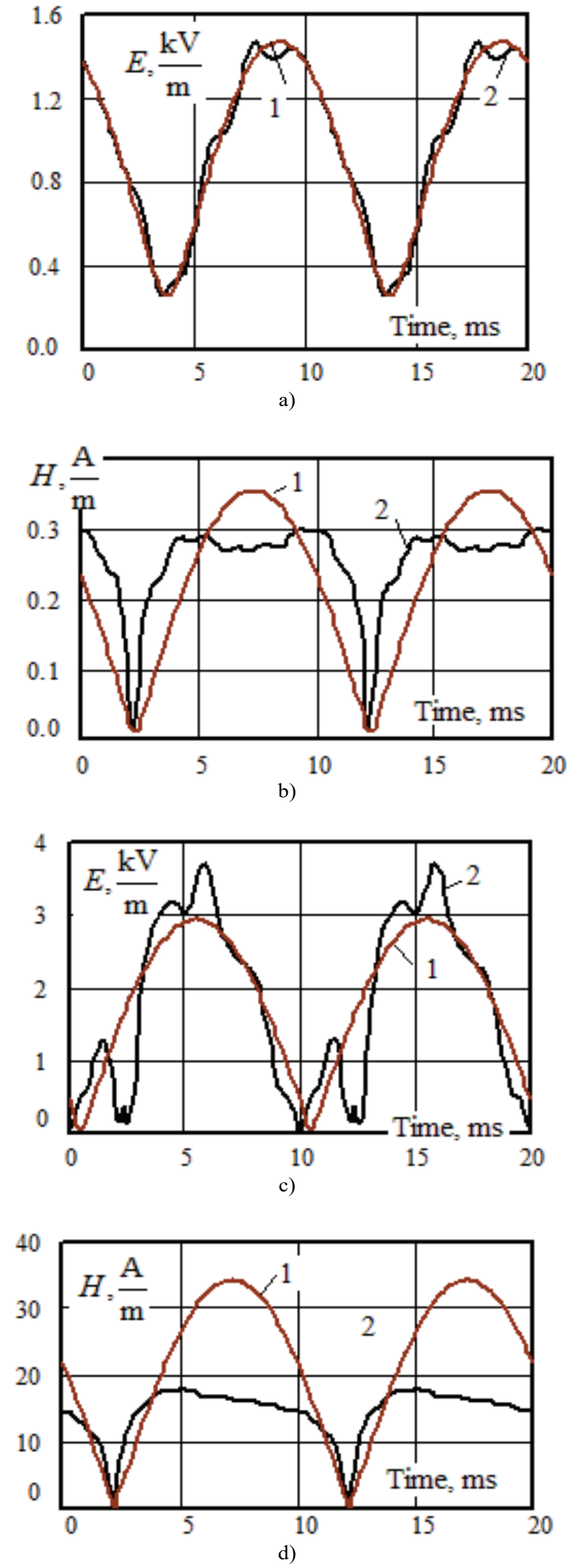

Fig. 27. Shapes of intensity curves at the 206-th simulation minute: $a, b-O P L ; c, d$-traction network; $a, c$-electrical field; $b, d$-magnetic field. the following relationship:

$$
\begin{aligned}
& k \omega t_{k \min , \max }=-\frac{1}{2} \operatorname{arctg}\left(\left(E_{k m x}^{2} \sin 2 \psi_{k x}+\right.\right. \\
& \left.\left.+E_{k m y}^{2} \sin 2 \psi_{k y}\right) /\left(E_{k m x}^{2} \cos 2 \psi_{k x}+E_{k m y}^{2} \cos 2 \psi_{k y}\right)\right) .
\end{aligned}
$$

The first arctangent value is chosen within $[-\pi / 2, \pi / 2]$, the second one differs by the value of $\pi$. The maximum and minimum absolute values of intensity are determined by the signs of the second derivative:

$$
\begin{aligned}
& \operatorname{sign}\left(E_{k m x}^{2} \cos \left[2\left(k \omega t_{k \max }+\psi_{k x}\right)\right]+\right. \\
& \left.+E_{k m y}^{2} \cos \left[2\left(k \omega t_{k \max }+\psi_{k y}\right)\right]\right) .
\end{aligned}
$$

The directions of the vector maxima and minima $\vec{E}(t)$ vary within the period according to the changes in the direction cosines

$$
\begin{aligned}
& \cos \left(\vec{e}_{i}, \vec{E}_{k \text { max }}\right)=\frac{E_{k m i} \sin \left(k \omega t_{\max }+\psi_{k i}\right)}{E_{k \text { max }}} ; \\
& E_{k \text { max }}=\sqrt{\sum_{i=1}^{2}\left[E_{k m i} \sin \left(k \omega t_{\text {max }}+\psi_{k i}\right)\right]^{2}} ; \\
& \cos \left(\vec{e}_{i}, \vec{E}_{k \text { min }}\right)=\frac{E_{k m i} \sin \left(k \omega t_{\text {min }}+\psi_{k i}\right)}{E_{k \text { min }}} ; \\
& E_{k \text { min }}=\sqrt{\sum_{i=1}^{2}\left[E_{k m i} \sin \left(k \omega t_{\text {min }}+\psi_{k i}\right)\right]^{2}},
\end{aligned}
$$

where $i=x, y$.

The horizontal and vertical components of the magnetic field intensity of the multi-wire line at harmonics are calculated using the formulas similar to those given above:

$$
\begin{aligned}
& \dot{H}_{k x}=\frac{1}{2 \pi} \sum_{i=1}^{N} \dot{I}_{k i} \frac{y-y_{i}}{\left(x_{i}-x\right)^{2}+\left(y_{i}-y\right)^{2}} ; \\
& \dot{H}_{k y}=-\frac{1}{2 \pi} \sum_{i=1}^{N} \dot{I}_{k i} \frac{x-x_{i}}{\left(x_{i}-x\right)^{2}+\left(y_{i}-y\right)^{2}},
\end{aligned}
$$

with the only difference, the effective value of current $\dot{\mathrm{I}}_{k i}$ of wire $i$ is taken for harmonic $k$.

These formulas are valid for all harmonics of practical importance at distances of no more than $100 \mathrm{~m}$ from a system of wires that create the field. Parameters of the magnetic field intensity vector are determined in the same way as for the electric field.

The presented technique is implemented in the Fazonord software. The simulation assumes the following:

- the electric system under study may contain transmission lines, transformers, loads, and AC traction network;

- the earth surface is assumed to be flat and perfectly conductive for the electric field; image charges are used to factor in the earth influence; the magnetic field of the near area is virtually not affected by the earth; however, the Carson formulas [28] are implemented in the software to calculate EMF at distances of more than $100 \mathrm{~m}$ from the field source;

- based on the network calculation, the wire potentials and currents are determined; electric field intensities are calculated via the linear charges of wires with the help of potential coefficients; 
Table 1. Maximum and average values of EMF intensity amplitudes for OPL and Traction Network.

\begin{tabular}{|c|c|c|c|c|c|}
\hline Network element & Field & Index & $\begin{array}{l}\text { Higher harmonics } \\
\text { considered }\end{array}$ & $\begin{array}{c}\text { Higher harmonics are } \\
\text { not considered }\end{array}$ & Difference, $\%$ \\
\hline \multirow{4}{*}{ OPL } & \multirow{2}{*}{ Electric, kV/m } & Average & 1.47 & 1.47 & 0.0 \\
\hline & & Maximum & 1.58 & 1.48 & 6.8 \\
\hline & \multirow{2}{*}{ Magnetic, A/m } & Average & 0.32 & 0.37 & -13.5 \\
\hline & & Maximum & 0.37 & 0.98 & -15.3 \\
\hline \multirow{4}{*}{ Traction network } & \multirow{2}{*}{ Electric, $\mathrm{kV} / \mathrm{m}$} & Average & 3.62 & 3.14 & 15.3 \\
\hline & & Maximum & 3.14 & 3.52 & 8.2 \\
\hline & \multirow{2}{*}{ Magnetic, A/m } & Average & 13.8 & 14.9 & -7.4 \\
\hline & & Maximum & 37.4 & 43.4 & -13.8 \\
\hline
\end{tabular}

- EMF intensities are calculated for each harmonic. An enlarged algorithm of EMF calculation in terms of harmonics includes the following stages.

1. Generate a calculation diagram, which includes models of multi-wire lines and transformers connected to other elements.

2. Set the coordinates of space points for each line under study. These space points require the intensities to be calculated.

3. Calculate EMF intensities for each instantaneous diagram after determining the operating conditions at the frequency of the current harmonic, including the fundamental one, with the above formulas:

- Calculate potential coefficients;

- Determine complexes of linear charge densities of wires;

- Determine components of electric field intensity complexes at the control points of space;

- Calculate currents of individual wires of multiwire systems and magnetic field intensities at the same points.

4. Determine time dependences of total field intensities of harmonics with the maximum values obtained for a time interval equal to the period of the fundamental frequency. Intensity curve shapes are calculated with a $0.2 \mathrm{~ms}$ step. Based on these data and the scale selected, the vector loci are plotted.

A typical diagram of traction and external AC power supply system is chosen to illustrate the capabilities of the technique described. It includes four sections of 220 $\mathrm{kV}$ OPL with AC-240 wires, three traction substations, and two inter-substation zones (ISZ) with a length of $50 \mathrm{~km}$. The EMF intensities, given harmonics, were calculated in the middle of the left-hand ISZ and on the OPL left-hand end adjacent to the intermediate traction substation. The sources of current harmonics were seven trains of odd direction with a weight of 6300 tons and seven trains of even direction with a weight of 6000 tons (Fig. 19). The mountain pass section of the railroad with the pass at the railway milepost 219 $\mathrm{km}$ entails considerable current consumption by trains (Fig. 20). The EMF calculations require, as input data, coordinates of the multi-wire system wires that are equivalent in terms of sag. Fig. 21 shows their crosssectional position.
Simulation modeling of train operation at the interval of simulated time up to the 327 th min provided the time dependences of the following parameters:

- total harmonic voltage and current distortion factors in the middle of the left-hand ISZ, and on the left-hand end of OPL of the second substation; Fig. 22 shows the dependences at the interval from the 100th to the 200th minute, when the railroad section is filled with trains;

- maximum values of electric and magnetic field intensities at the height of $1.8 \mathrm{~m}$ (Figs. 23, 24); the intensities were calculated for the traction network at the point with coordinates $x=2.1 \mathrm{~m} ; y=1.8 \mathrm{~m}$; for OPL $x=4 \mathrm{~m} ; y=1.8 \mathrm{~m}$.

The time dependences of $k_{U}, k_{I}$ parameters, shown in Fig. 22, indicate that the operating conditions under consideration are characterized by significant levels of harmonic current and voltage distortions. The maximum values of $k_{U}, k_{I}$ for the traction network reach 60 and $75 \%$, respectively, and for the OPL - 14 and $55 \%$, for the whole simulation time. The overhead catenary of the odd track is characterized by higher levels of harmonics, which is associated with high traction loads of the odd track close to the control point.

Figures 22, 23, and 24 demonstrate significant variability of non-sinusoidality and EMF intensity parameters associated with trains operation. The maximum amplitudes of the OPL electric field intensities, calculated for higher harmonics, are higher than similar indicators for the field of fundamental frequency by $7 \%$ (Table 1). However, a reverse situation is observed for the magnetic field - the intensities maxima decrease by $15 \%$ (with higher harmonics considered), which is associated with the specific features of phase characteristics of current harmonics.

The loci of EMF intensity vectors indicate the ellipse distortions due to the higher harmonic current and voltage effect. The same distortions are observed in the shapes of curves of the field absolute values during the period of fundamental frequency (Fig. 27). The presented results confirm the importance of factoring in harmonic distortions when simulating EMF generated by traction networks and power lines adjacent to traction substations.

The presented technique of calculating the intensities of EMF generated by power lines and traction networks includes preliminary computations of operating conditions 
of the integrated system of traction and external power supply at fundamental and harmonic frequencies as an essential stage. The considered example of a three-phase single-phase electrical network has proved the importance of considering harmonic distortions when determining EMF. This technique can also be employed to factor in higher armonics to determine EMF generated by multiwire power lines of any design.

\section{CONCLUSION}

The technique developed to determine electromagnetic fields generated by overhead power lines has the following features:

1. systems approach, which manifests itself in the possibility of simulating electromagnetic fields considering the properties and characteristics of a complex TPSS and a supplying EPS;

2. universality ensuring simulation of power transmission lines and traction networks of various designs;

3. appropriateness to the environment, which is achieved via a precise analysis of the underlying surface, underground communications, and artificial railroad structures, such as galleries, bridges, and tunnels;

4. comprehensiveness provided by integrating the network calculation and the EMF intensity determination at the fundamental frequency and higher harmonics frequencies.

In the context of power industry digitalization, this technique, when put into practice, will provide the scientifically grounded approach to the analysis of electromagnetic safety conditions in electric power systems and at railroad transport facilities and the development of measures to improve electromagnetic safety.

\section{REFERENCES}

[1] S.M. Apollonsky, A.N. Bogarinova, "EMF intensities of the air environment on the electrified railroad," Collection of reports of the ninth Russian research and technology conference on electromagnetic compatibility of technical means and electromagnetic safety. St.P., Russian, 2006.

[2] A.I. Sidorov, I.S. Okrainskaya, Electromagnetic fields near ultrahigh voltage electrical installations. Chelyabinsk: South Ural State University, 2008, p. 204.

[3] A.B. Kosarev, B.I. Kosarev, Basics of electromagnetic safety of railroad transport power supply systems. Moscow: Intext, 2008, p. 480.

[4] N.V. Byakova, V.P. Zakaryukin, A.V. Kryukov, Electromagnetic safety in railroads power supply systems: modeling and control: monograph, ed. by A.V. Kryukov. Angarsk: Editorial and Publishing Department, Angarsk State Technical University, 2018, p. 382.

[5] Levitt B. Blake, Protection against electromagnetic fields. On the effects of household appliances, cell phones, power lines and other electrical devices on the human body. Moscow: ACT, Astel, 2007, p. 447.

[6] Lars Abrahamsson, Railway Power Supply Models and Methods for Long-term Investment Analysis. Licentiate Thesis. Royal Institute of Technology School of Electrical Engineering Electric Power Systems. Stockholm, Sweden, 2008, p. 166.

[7] R. Doleček, O. Černý, Z. Němec and J. Pidanič, “The behavior of the traction power supply system of AC 25 kV 50 Hz during operation," Rail Transport-Systems Approach, Studies in Systems, Decision and Control 87. Springer International Publishing AG, 2017, pp. 35-65.

[8] A. Ogunsola and A. Mariscotti, Electromagnetic Compatibility in Railways. Springer-Verlag Berlin Heidelberg 2013, p. 568.

[9] M. Mandić, I. Uglešić, V. Milardić, "Design and testing of $25 \mathrm{kV}$ AC electric railway power supply systems," Tehnički vjesnik 20, pp. 505-509, March 2013.

[10] R.J. Hill, "Modelling and simulation of electric railway traction, track signaling and power systems," Transactions on the Built Environment. Vol. 6, pp. 383-390, 1994.

[11] Luan Xiaotian, Zhu Haijing, Qiu Bo, Han Bochong, "EMC in Rail Transportation," CUE2016-Applied Energy Symposium and Forum 2016: Low carbon cities \& urban energy systems. Available at: www. sciencedirect.com. (accessed 30.04.2021)

[12] S. Baranowski, H. Ouaddi, L. Kone and N. Idir, "EMC Analysis of Railway Power Substation Modeling and Measurements Aspects," Infrastructure Design, Signalling and Security in Railway. ISBN: 978-95351-0448-3, InTech. Available at: www.intechopen. com. (accessed 30.04.2021)

[13] H. Ouaddi, S. Baranowski, G. Nottet, B. Demoulin, and L. Koné. (2011). Study of HF behaviour of railway power substation in reduced scale. The European Physical Journal - Applied Physics, 53(3), 33603. DOI:10.1051/epjap/2010100061

[14] $\mu$ PAS. Power Application Software. User manual for dynamic train-drive simulation. PROLEITEC AG, CH-8903 Birmensdorf, February 2013. p. 167.

[15] Fabel - Enotrac Ag, Software for the simulation of electric vehicles and power supply networks. Available at: www.enotrac.com. (accessed 30.04.2021)

[16] R. Kircher, J. Klühspies, R. Palka, et al. "Electromagnetic Fields Related to High Speed Transportation Systems," Transportation Systems and Technology 2018, vol. 4(2), pp. 152-166. DOI: 10.17816 / transsyst201842152-166.

[17] R. Kircher, R. Palka, E. Fritz, K. Eiler, M. Witt, L. Blow, J. Klühspies, "Electromagnetic Fields of High-Speed Transportation Systems Maglev Technologies in Comparison with Steel-WheelRail," The International Maglev Board C/O CERM Institute, Technical University of Deggendorf of Applied Sciences. Available at: www.maglevboard. net. (accessed 30.04.2021) 
[18] V.P. Zakaryukin, A.V. Kryukov, Complex asymmetrical modes of electrical systems. Irkutsk: Irkutsk State University, 2005. 273 p.

[19] V.P. Zakaryukin, A.V. Kryukov, A.V. Cherepanov Intelligent technologies of power quality management. Irkutsk: ISTU Publishing House, 2015. p. 218.

[20] O.I. Mitruyev, "Phase coordinate method to compute industrial frequency electromagnetic fields," Enhancing energy production efficiency and use in the conditions of Siberia. Irkutsk: ISTU Publishing House. 2006. pp. 301-311.

[21] R.A. Kats, L.S. Perelman, "Computation of electrical field of a three-phase power line," Elektrichestvo, no. 1, pp. 16-19, 1978.

[22] G.A. Greenberg, B.E. Bonstedt, "Fundamentals of the exact theory of the wave field of power lines," Journal of Theoretical Physics, vol. 24, no. 1, pp. 7-95, 1954.

[23] M.V. Kostenko, D.G. Messerman, "Deformation of lightning overvoltage waves in over and ultra high voltage transmission lines at long run lengths," Proceedings of the Academy of Sciences of the USSR: Energy and Transport, no. 3, pp. 158-164. 1987.

[24] D.G. Messerman, L.S. Perelman, "Parameters of wave propagation along a long wire above the ground," Proceedings of the Academy of Sciences of the USSR. Energy and Transport, no. 1, pp. 65-74, 1986.

[25] D.G. Messerman, L.S. Perelman, "Calculation of modal parameters of multi-wire power transmission lines," Proceedings of the Academy of Sciences of the USSR. Energy and Transport, no. 1, pp. 9-17, 1984.

[26] L.M. Wedepohl, “Application of matric methods to the solution of travelling-wave phenomena in polyphase systems," IEEE, vol. 110, no. 12, 1963.

[27] L.M. Wedepohl, "Electrical characteristics of polyphase transmission systems with special reference to boundary-value calculations at power-line carrier frequencies," IEEE, vol. 112, no. 11, 1965.

[28] I.R. Carson, "Wave propagation in overhead wires with ground return," Bell System Technical Journal, vol. 5, pp. 539-554, 1926.

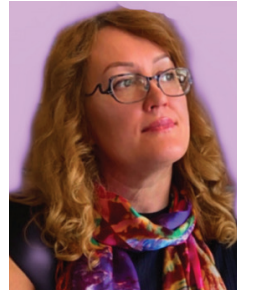

Natalya Buyakova is an Associate Professor in the Department of Power Supply of Industrial Enterprises, Angarsk State Technical University. In 1995, she graduated from Angarsk Technological Institute. Natalya Buyakova received the Ph.D. degree in engineering from Irkutsk State University of Transport in 2012. Her research interests are modeling and management of electric power systems and railroad supply systems; and smart grids.

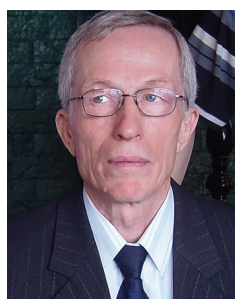

Vasili Zakaryukin is Consultant Professor at LLC "Smart grid" at Irkutsk State Transport University. He graduated from Irkutsk State University in 1971. Vasili Zakaryukin received the degree of Ph.D. in engineering from Tomsk Polytechnic Institute in 1983 and the degree of D.Sc. in engineering from Omsk State Transport University in 2010. His research interests are modeling of electric power systems and railway power supply systems.

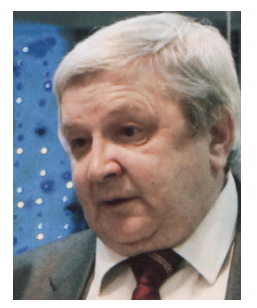

Andrey Kryukov is a Professor at Transport Power Engineering Department at Irkutsk State Transport University, a Professor at Power Supply and Electrical Equipment Department at Irkutsk National Research Technical University, Russia. He graduated from East Siberian Technological Institute in 1974. Andrey Kryukov received his degree of Ph.D. in engineering from Leningrad Polytechnic Institute in 1982 and the degree of D.Sc. in engineering from Energy Systems Institute of the Russian Academy of Sciences in 1997. His research interests are modeling and control of electric power systems and railway power supply systems; and smart grids.

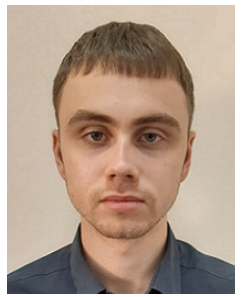

Dmitry Seredkin is a postgraduate student at Irkutsk State Transport University. In 2019, he graduated from Irkutsk State Transport University, majoring in Railway Power Supply. His research interests are modeling of electric power systems, electromagnetic safety, and electromagnetic compatibility. 